\title{
Cancer vaccine formulation dictates synergy with CTLA-4 and PD-L1 checkpoint blockade therapy
}

\author{
Yared Hailemichael, ${ }^{1}$ Amber Woods, ${ }^{2}$ Tihui Fu, ${ }^{3}$ Qiuming He, ${ }^{3}$ Michael C. Nielsen, ${ }^{1}$ Farah Hasan, ${ }^{1}$ Jason Roszik, ${ }^{1}$ Zhilan Xiao, ${ }^{1}$ \\ Christina Vianden, ${ }^{1}$ Hiep Khong, ${ }^{1}$ Manisha Singh, ${ }^{1}$ Meenu Sharma, ${ }^{1}$ Faisal Faak, ${ }^{1}$ Derek Moore, ${ }^{1}$ Zhimin Dai, ${ }^{1}$ Scott M. Anthony, ${ }^{4}$ \\ Kimberly S. Schluns, ${ }^{4,5}$ Padmanee Sharma, ${ }^{3}$ Victor H. Engelhard, ${ }^{2}$ and Willem W. Overwijk ${ }^{1,5}$ \\ 'Department of Melanoma Medical Oncology, The University of Texas MD Anderson Cancer Center, Houston, Texas, USA. ²Department of Microbiology, Immunology, and Cancer Biology, \\ Carter Immunology Center, University of Virginia School of Medicine, Charlottesville, Virginia, USA. ${ }^{3}$ Department of Genitourinary Medical Oncology/Immunology and ${ }^{4}$ Department of Immunology, \\ The University of Texas MD Anderson Cancer Center, Houston, Texas, USA. ${ }^{5}$ The University of Texas Graduate School of Biomedical Sciences at Houston, Houston, Texas, USA.
}

\begin{abstract}
Anticancer vaccination is a promising approach to increase the efficacy of cytotoxic T lymphocyte-associated protein 4 (CTLA-4) and programmed death ligand 1 (PD-L1) checkpoint blockade therapies. However, the landmark FDA registration trial for anti-CTLA-4 therapy (ipilimumab) revealed a complete lack of benefit of adding vaccination with gp100 peptide formulated in incomplete Freund's adjuvant (IFA). Here, using a mouse model of melanoma, we found that gp100 vaccination induced gp100-specific effector T cells (Teffs), which dominantly forced trafficking of anti-CTLA-4-induced, non-gp100-specific Teffs away from the tumor, reducing tumor control. The inflamed vaccination site subsequently also sequestered and destroyed anti-CTLA-4-induced Teffs with specificities for tumor antigens other than gp100, reducing the antitumor efficacy of anti-CTLA-4 therapy. Mechanistically, Teffs at the vaccination site recruited inflammatory monocytes, which in turn attracted additional Teffs in a vicious cycle mediated by IFN- $\gamma$, CXCR3, ICAM-1, and CCL2, dependent on IFA formulation. In contrast, nonpersistent vaccine formulations based on dendritic cells, viral vectors, or water-soluble peptides potently synergized with checkpoint blockade of both CTLA-4 and PD-L1 and induced complete tumor regression, including in settings of primary resistance to dual checkpoint blockade. We conclude that cancer vaccine formulation can dominantly determine synergy, or lack thereof, with CTLA-4 and PD-L1 checkpoint blockade therapy for cancer.
\end{abstract}

\section{Introduction}

Therapeutic blockade of the checkpoint receptors cytotoxic $\mathrm{T}$ lymphocyte associated protein 4 (CTLA-4) and programmed cell death protein 1 (PD-1) on T cells can cure patients with metastatic cancer (1-3). However, many patients do not experience a clinical benefit, and significant hurdles remain in increasing the therapeutic benefit of checkpoint blockade (4-7). Recent evidence points to poor preexisting antitumor $\mathrm{T}$ cell immunity $(8,9)$ and genomic defects in IFN- $\gamma$ pathway genes in tumor cells (10) as a cause for primary resistance to CTLA- 4 checkpoint blockade therapy. One strategy to increase tumor-specific $\mathrm{T}$ cell immunity is vaccination (11), and consequently, vaccination is being explored as an avenue to increase clinical efficacy of anti-CTLA-4 and anti-PD-1 therapies (12).

However, the same landmark study (1) of 676 patients with melanoma that led to FDA approval of anti-CTLA-4 (ipilimumab, Yervoy) showed no therapeutic enhancement of anti-CTLA-4 monotherapy by concurrent vaccination with gp100 peptide in incomplete Freund's adjuvant (IFA). Instead, addition of gp100/ IFA vaccination significantly decreased the best overall response rate $(P=0.04)$ and disease control rate $(P=0.04)$ to ipilimumab,

Conflict of interest: The authors have that declared that no conflict of interest exists Submitted: February 8, 2017; Accepted: January 9, 2018.

Reference information: / Clin Invest. 2018;128(4):1338-1354.

https://doi.org/10.1172/JCI93303. suggesting a surprising, dominant-negative effect of the vaccine on the efficacy of CTLA-4 checkpoint blockade (1). These results leave uncertain how best to combine vaccination with checkpoint blockade to increase therapeutic efficacy.

Currently there are 33 registered clinical trials in the USA alone (according to ClinicalTrials.gov) in which a cancer vaccine is combined with anti-CTLA-4 and/or anti-PD-1 checkpoint blockade (Supplemental Table 1; supplemental material available online with this article; https://doi.org/10.1172/JCI93303DS1). Of these trials, initiated by a variety of academic and industry parties in a broad range of cancers, 14 (42\%) use an IFA-based vaccine, indicating how widely this formulation is applied clinically. Our data suggest that in these trials the addition of IFA-based vaccine is likely to result in no additional, and possibly reduced, clinical benefit of checkpoint blockade therapy. Conversely, our results suggest that switching to a different vaccine formulation may result in strongly increased therapeutic efficacy. These findings may have direct implications for current and future patients treated with these combination regimens.

We previously reported (13) that vaccination with gp100 peptide in IFA creates a persisting antigen depot that primes antigenspecific $\mathrm{CD}^{+} \mathrm{T}$ cells, which is followed by the undesirable sequestration of these cells at the vaccination site, and eventually their exhaustion and apoptosis, resulting in negligible antitumor activity. Similar results have been observed in patients with melanoma after vaccination with multiple melanoma antigen-derived pep- 


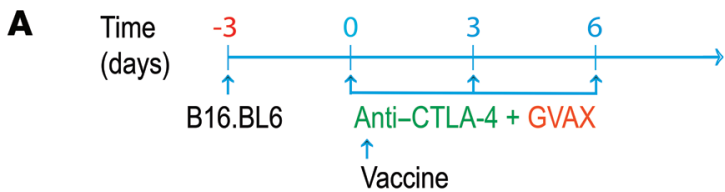

\section{$B$}

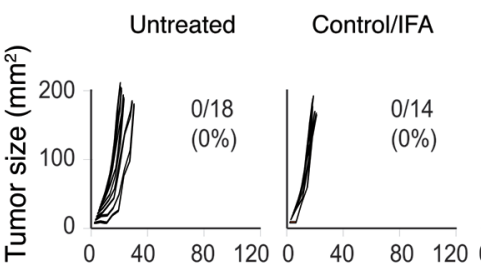

\begin{abstract}
Gp100/IFA
\end{abstract}
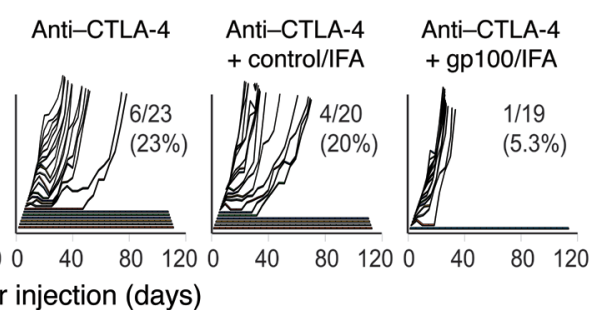

C

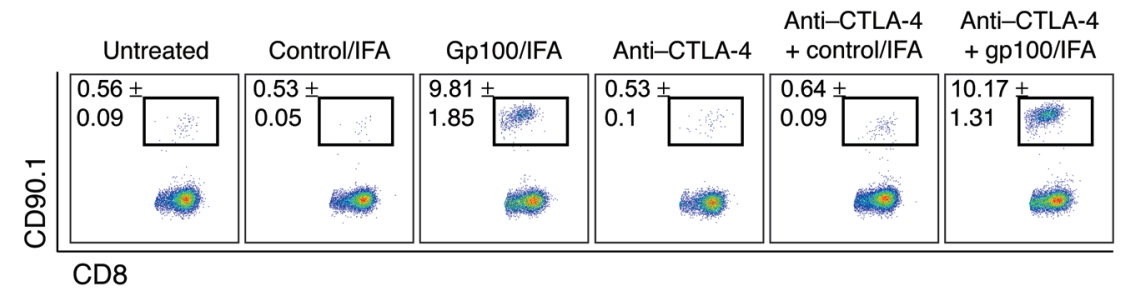

D

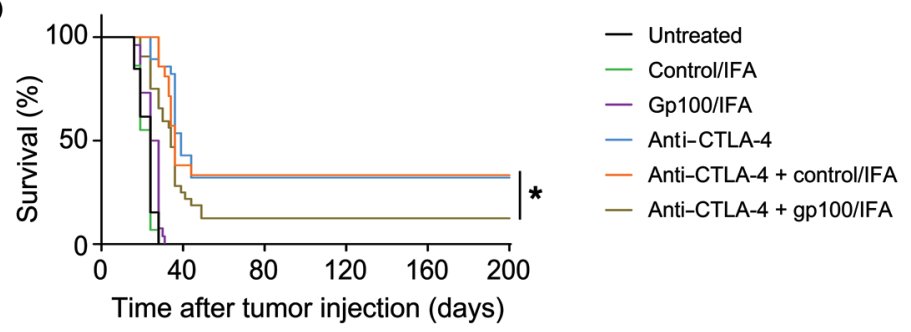

Figure 1. Anti-CTLA-4 therapeutic activity is impaired by the addition of gp100/IFA vaccination. (A) Experimental scheme. (B) Mice bearing 3-day-old B16 tumors received naive gp100-specific CD90.1+ pmel-1 $T$ cells i.v., followed by s.c. vaccination with hgp100/IFA or control/IFA on day 0 and/or anti-CTLA-4 plus Gvax therapy on days 0,3 , and 6 , or were left untreated. Tumor size in individual mice is shown. Numbers in parentheses represent the proportion of mice with tumor-free survival. (C) pmel-1 T cells in PBMCs 7 days after vaccination. Data represent mean \pm SEM, $n=5$. (D) Kaplan-Meier survival curves. Data are pooled from 2 independent experiments, each with 8 to 15 mice per group. tides formulated in IFA (14). Here we test the hypothesis that the inflamed, chemokine-rich vaccination site also potently sequesters the systemic pool of checkpoint blockade-induced effector $\mathrm{T}$ cells with vaccine-unrelated tumor antigen specificity, reducing the efficacy of CTLA-4- and PD-1-based checkpoint blockade therapy. We subsequently demonstrate that nonpersisting, virus, or water-based vaccines do not induce these deleterious effects and instead potently synergize with CTLA-4 and PD-1 checkpoint blockade therapy.

\section{Results}

Anti-CTLA-4 therapeutic activity is impaired by addition of gp100/ IFA vaccination. To understand the parameters that control synergy between checkpoint blockade and anticancer vaccination, we modeled the clinical scenario of anti-CTLA-4 therapy of melanoma plus vaccination with gp100 peptide in IFA (1) by adapting the standard mouse model of anti-CTLA-4 therapy of B16 melanoma (15-17) to include vaccination with gp100 ${ }_{25-33}$ peptide in IFA. To correct for the fact that B16 melanoma progresses so rapidly that there is no time to raise gp100-specific effector T cells (Teffs) by multiple cycles of vaccination as given to the patients (1), we adoptively transferred naive, gp100-specific T cell receptor (TCR) transgenic pmel- $1\left(\mathrm{Vb}_{3}{ }^{+} \mathrm{CD} 90.1^{+} \mathrm{CD} 8^{+}\right) \mathrm{T}$ cells. The pmel-1 T cell recognizes the gp100 ${ }_{25-33}$ peptide derived from the murine gp100 protein encoded by the pmel-17 gene, an ortholog of the melanocyte differentiation antigen gp100, which is often overexpressed in human melanomas and recognized by human $\mathrm{CD}^{+} \mathrm{T}$ cells (18, 19). No untreated mice or mice receiving a mock vaccine (control/ IFA), or gp100 peptide in IFA (gp100/IFA) were cured, whereas $23 \%$ of the animals were cured with anti-CTLA- 4 therapy (Figure 1 , $A$ and $B$ ). The addition of control/IFA vaccination to anti-CTLA-4 therapy did not affect the therapeutic impact. Interestingly, paralleling the observations in patients (1), gp100/IFA vaccination did not enhance, but significantly decreased, the therapeutic efficacy of anti-CTLA-4 therapy despite inducing a high level of gp100-specific CD8 ${ }^{+}$Teffs in peripheral blood leukocytes (PBLs) (Figure 1, C and D, $P<0.0001$ ). Thus, gp100/IFA-based vaccination reduced the therapeutic efficacy of anti-CTLA-4 therapy. To both extend and replicate our finding beyond B16 melanoma, we treated E.G7.OVA, a thymoma-expressing OVA, as a model antigen. We found that the addition of $\mathrm{OVA}_{257-264}$ peptide in IFA (OVA/ IFA) immunization to primed endogenous OVA-specific $T$ cells significantly impaired anti-CTLA-4-induced therapeutic activity (7\% of mice cured) compared with anti-CTLA-4 plus control/ IFA (27\%) or anti-CTLA-4 monotherapy (60\%), OVA/IFA (0\%), control/IFA (0\%), or no treatment (0\%) (Supplemental Figure 1, $\mathrm{A}-\mathrm{C} ; \mathrm{P}<0.0001)$. This finding suggests that vaccination-induced loss of anti-CTLA-4 activity was not a peculiarity of the B16 tumor model, of pmel-1 T cells, or of gp100 peptide as a vaccine antigen.

$C D 8^{+}$Teffs localize to sites of vaccination with noncognate antigen. Vaccination with gp100/IFA sets up a persistent, chronically 
A
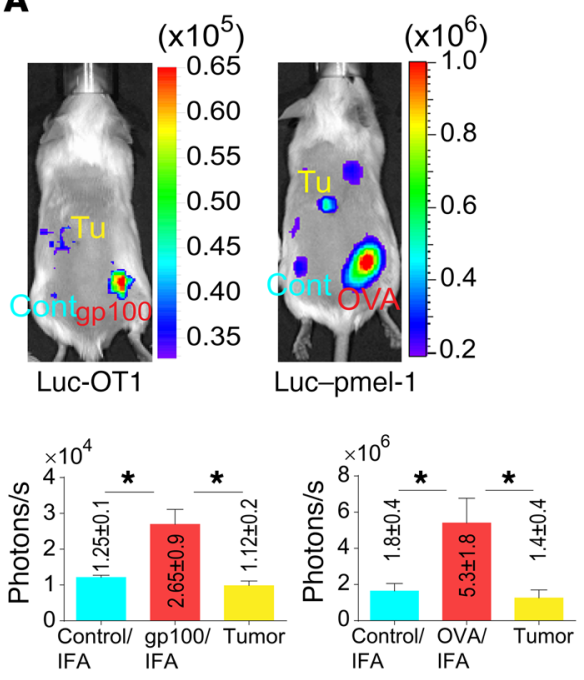

E
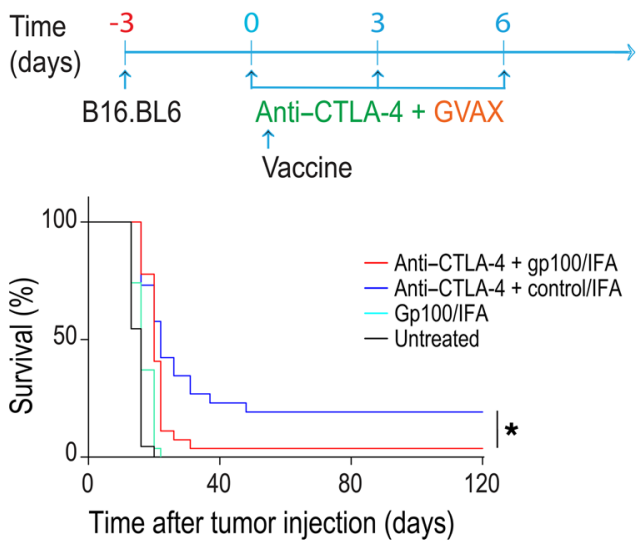

B
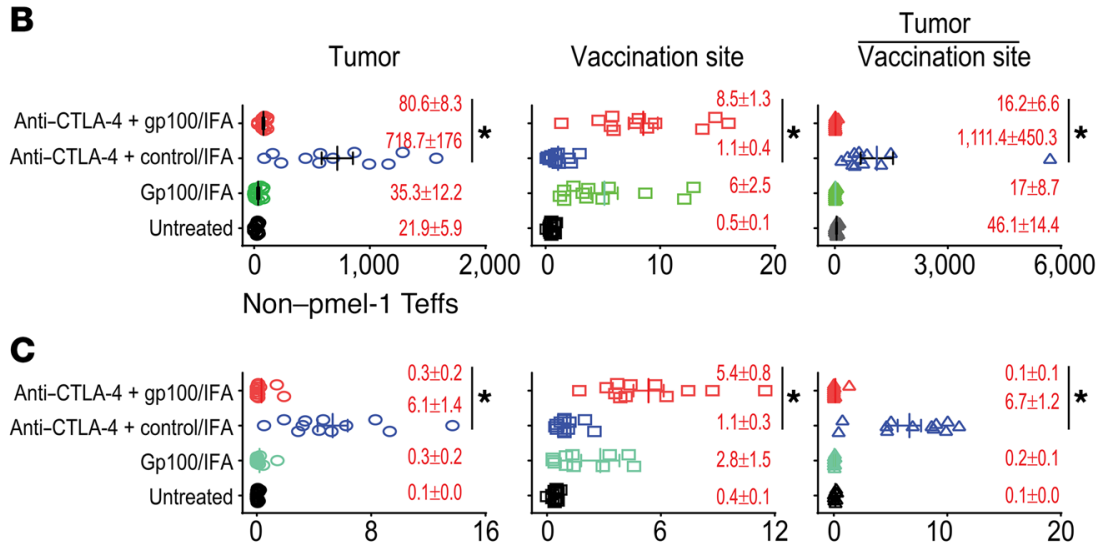

TRP-2-specific Teffs

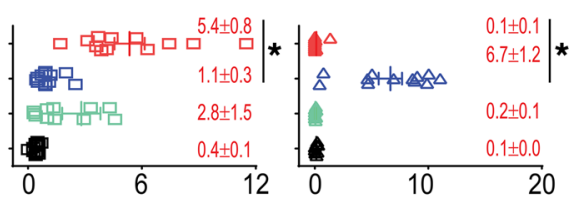

D

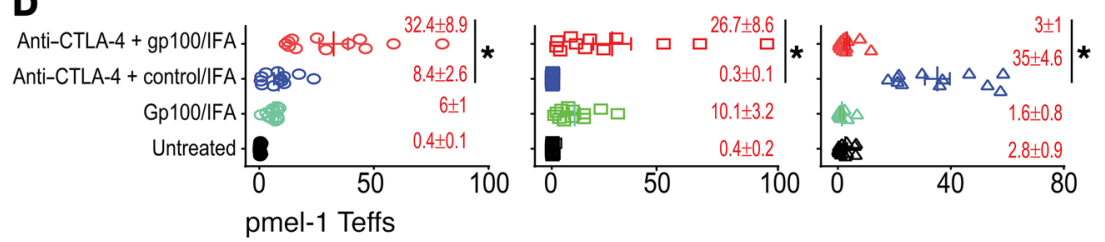

Figure 2. CD8+ Teffs localize to sites of vaccination with noncognate antigen. (A) Mice bearing 7-day-old, s.c. B16 tumors received 6-day-cultured, gp100-specific pmel-1 or OVA-specific OT-1 Teffs i.v., followed by vaccination with hgp100/IFA (right flank, s.c.) or OVA/IFA (right flank, s.c.). v-effLuctransduced OT-1 T cells (left panel) and v-effLuc-transduced pmel-1 T cells (right panel) were visualized by whole-mouse imaging 4 days after vaccination. Color bars represent mean \pm SEM photons/second $\left(n=5,{ }^{*} P<0.05\right)$ determined by 1 -way ANOVA with a post hoc test. Statistical differences between the 2 groups were determined by the unpaired 2-tailed $t$ test. (B-E) Mice bearing 3-day-old, s.c. B16 melanoma received naive CD90.1+ pmel-1 T cells, early anti-CTLA-4 therapy, and vaccination with control/IFA or hgp100/IFA. Tumor and vaccination sites were harvested 9 days after vaccination.

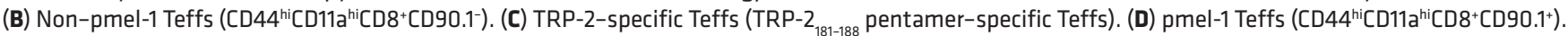
Absolute number counts were adjusted per tissue weight resection from tumor and vaccination sites. Data (B-D) represent mean \pm SEM of 3 independent experiments ( $n=5,{ }^{*} P<0.05$ unpaired 2-tailed $t$ test). (E) Experimental scheme (top) showing Kaplan-Meier survival curve data pooled from 3 independent experiments ( $n=5$ mice per group, bottom). ${ }^{*} P<0.05$, log-rank test.

inflamed antigen depot at the injection site, which sequesters gp100 vaccine-activated, gp100-specific Teffs, preventing them from reaching the tumor (13). We hypothesized that sequestration of Teffs at antigen-rich vaccine sites is not restricted to vaccine antigen-specific Teffs, but extends to other Teffs with specificities for antigens unrelated to those used in the vaccine. To test this hypothesis, we adoptively cotransferred luciferase-transduced OT-I Teffs and untransduced gp100-specific pmel-1 Teffs into mice vaccinated with gp100/IFA. Four days later, we observed strong accumulation of luciferase ${ }^{+}$OT-I Teffs at the gp100/IFA vaccination site, but minimal accumulation at the tumor or saline/ IFA control vaccination site (Figure $2 \mathrm{~A}, P<0.001)$. When we performed the converse experiment, more luciferase ${ }^{+}$pmel-1 Teffs accumulated at the $\mathrm{OVA}_{257-264}$ (SIINFEKL) peptide in the IFA vac- cination site than at the tumor or saline/IFA site (Figure 2A, $P<$ $0.001)$. Thus, specific $\mathrm{T}$ cell reactivity to persisting vaccine antigen induces sequestration and dysfunction of Teffs that recognize cognate vaccine antigen and also of Teffs with other antigenic specificities. Given our observation in Figure 2A, we wished to evaluate therapeutic activity of adoptively transferred OT-I T cells in mice with B16.OVA after gp100/IFA or control/IFA vaccination. We found that OT-I T cells in blood showed reduced proliferation, increased expression of inhibitory cell surface markers, and loss of therapeutic activity when a distant gp100/IFA vaccination site was present (Supplemental Figure 2, A-C).

We next studied the localization of anti-CTLA-4 therapyinduced Teffs during concurrent vaccination with gp100/IFA. As reported before, some of these Teffs recognized the melanocyte 
A

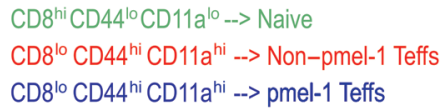

$\mathrm{CD} 8^{\mathrm{hi}} \mathrm{CD} 44^{10} \mathrm{CD} 11 \mathrm{a}^{10} \rightarrow \rightarrow$ Naive

$\mathrm{CD} 8^{10} \mathrm{CD} 44^{\text {hi }} \mathrm{CD} 11 \mathrm{a}^{\text {hi }} \rightarrow \rightarrow$ Non-pmel-1 Teffs

$\mathrm{CD} 8^{\circ} \mathrm{CD} 44^{\text {hi }} \mathrm{CD} 11 \mathrm{a}^{\text {hi }} \rightarrow \rightarrow$ pmel-1 1 Teffs

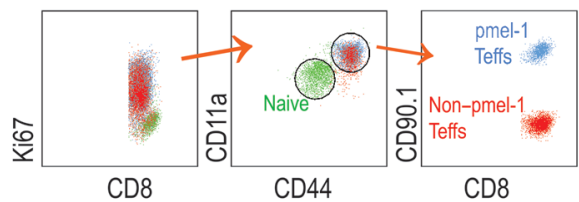

B

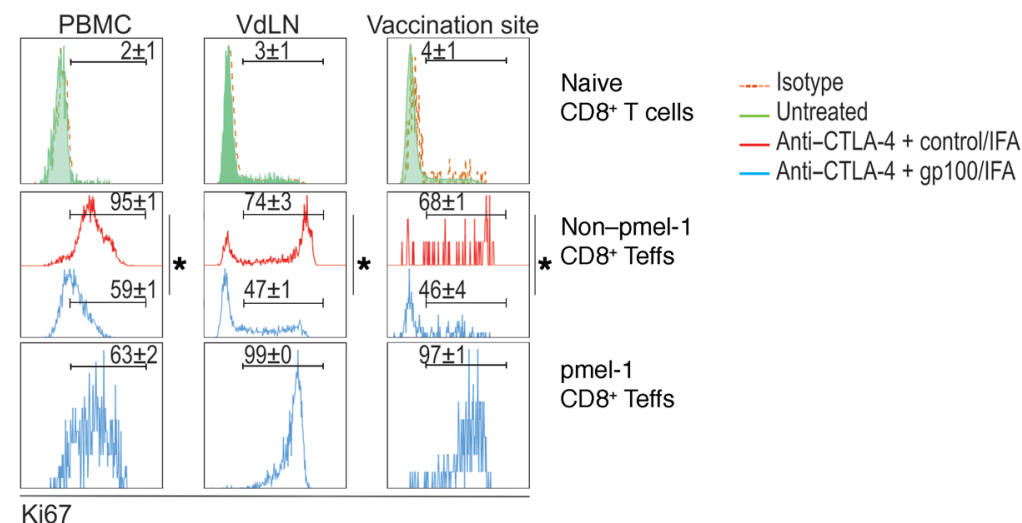

C

Anti-CTLA-4 + gp100/IFA

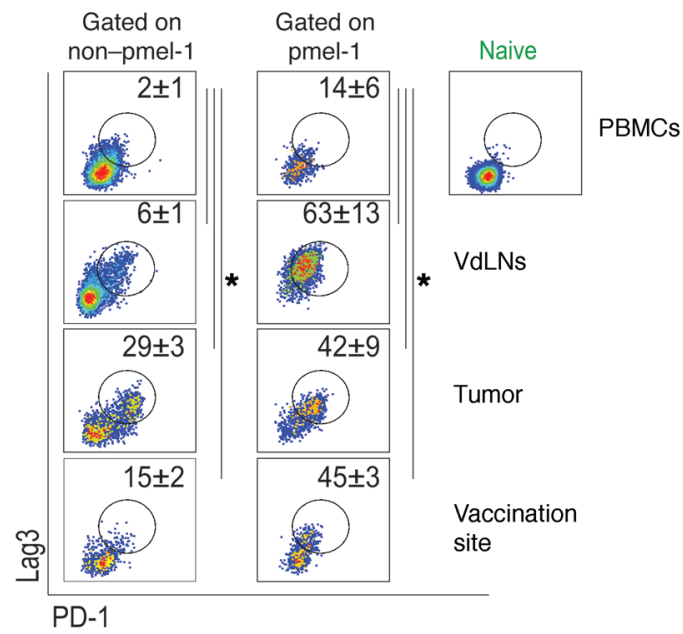

D Gated on non-pmel-1

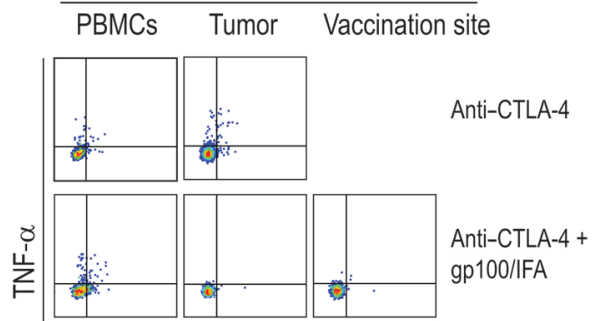

IFN- $\gamma$

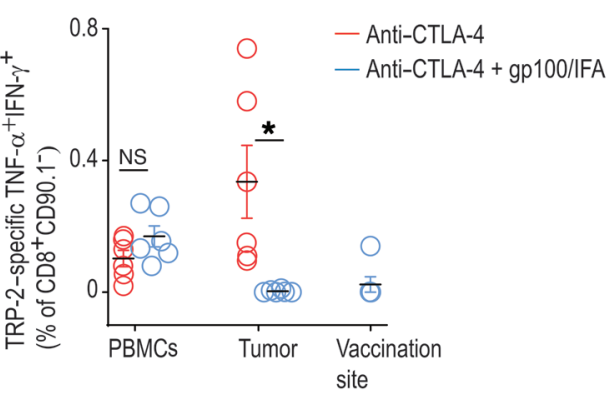

E

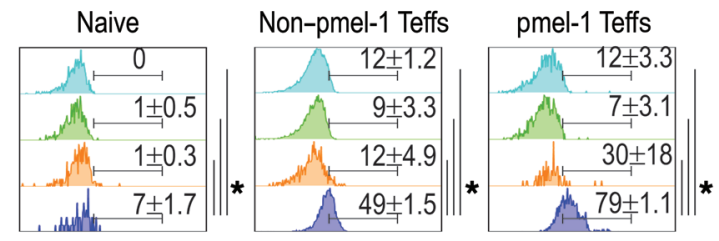

Fas
VdLNs

Spleen

Tumor

Vaccination site
F Naive Non-pmel-1 pmel-1

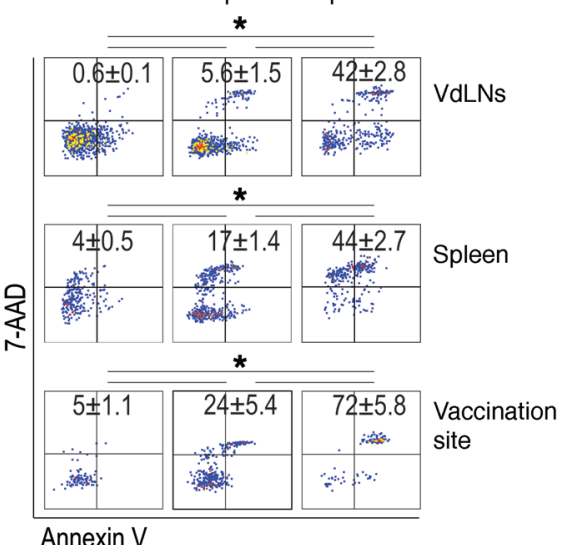

Figure 3. Exhaustion and apoptosis of sequestered anti-CTLA-4 activated CD8 ${ }^{+}$Teffs. (A) Gating strategy for naive, non-pmel-1 Teffs and pmel-1 Teffs from PBMCs (top). Mice bearing 3-day-old, s.c. B16 melanoma received CD90.1+ pmel-1 T cells, early anti-CTLA-4 therapy, and vaccination with control/ IFA or hgp100/IFA. (B) Ki67 protein expression by naive CD8 ${ }^{+}$T cells, non-pmel-1 CD8 ${ }^{+}$Teffs, and pmel-1 CD8 ${ }^{+}$Teffs in PBMCs, VdLNs, and at the vaccination site 9 days after the start of therapy. pmel-1 histograms show T cells in a gp100/IFA setting. (C) PD-1/LAG-3 expression in Teffs from PBMCs, VdLNs, tumor site, and vaccination site. (D) TRP-2-specific CD8 ${ }^{+}$Teffs in VdLNs (top panel) and quantitation of IFN- $\gamma^{+}$TNF- $\alpha^{+}$TRP-2-specific CD8 ${ }^{+}$Teffs in PBMCs, tumor site, and vaccination site (bottom panel). (E) Fas expression on naive T cells, non-pmel-1 Teffs, and pmel-1 Teffs from VdLNs, spleen, tumor site, and at the vaccination site 9 days after the start of therapy. (F) Apoptotic cell death from VdLNs, spleen, and at the vaccination site of naive CD8 ${ }^{+} T$ cells, non-pmel-1 CD8 ${ }^{+}$Teffs, and pmel-1 CD8 ${ }^{+}$Teffs 9 days after the start of therapy, as measured by flow cytometry of annexin V and 7-AAD staining. Plots are shown as mean \pm SEM ( $n=5,{ }^{*} P<0.05$ unpaired 2-tailed $t$ test). Data shown are representative of 2 experiments. 
differentiation antigen tyrosinase-related protein 2 (TRP-2) (15, 16); however, the majority of Teffs have unidentified specificities $(2,3,20)$. We therefore used a recently reported methodology to specifically detect the entire polyclonal Teff pool based on $\mathrm{CD} 8{ }^{\text {lo }} \mathrm{CD} 44^{\text {hi }} \mathrm{CD} 11 \mathrm{a}^{\text {hi }}$ surface expression (Supplemental Figure $3 \mathrm{~A})(21,22)$, as well as IFN- $\gamma$ secretion in response to specific antigen stimulation (Supplemental Figure 3B), and surveyed their localization at the tumor and vaccination sites 9 days after vaccination. Anti-CTLA-4 monotherapy markedly increased antiCTLA-4-induced non-pmel-1 CD8 ${ }^{+}$Teff number at the tumor site (Figure 2B). As expected, a fraction of these Teffs specifically recognized epitopes from the known murine melanoma antigens TRP-2 (Figure 2C), p15E (Supplemental Figure 4A), and gp100 (Supplemental Figure 4B). When gp100/IFA vaccination was added, the vast majority of anti-CTLA-4 therapy-induced noncognate (TRP-2 and p15E) antigen-specific and cognate (gp100) antigen-specific non-pmel-1 Teffs did not reach the tumor and instead colocalized with pmel-1 Teffs at the vaccination site (Figure 2, B-D, and Supplemental Figure 4, A and B). Thus, the combination therapy resulted in lower tumor/vaccination site ratios than CTLA-4 monotherapy for total non-pmel-1 Teffs (18:1 versus $1,366: 1, P=0.001)$, TRP-2-specific Teffs (0.3:5.4 versus 6:1, $P=0.005)$, p15E-specific Teffs (4:1 versus $86: 1, P=0.003)$, and non-pmel-1 gp100-specific Teffs (14:1 versus 29:1, $P=0.006$ ). Combination therapy also caused a significantly lower tumor/vaccination site ratio for gp100-specific pmel-1 Teffs (4:1 versus 36:1, $P=0.0004)$, resulting in reduced tumor control (Figure $2 \mathrm{E}$ ). The gp100/IFA vaccination site also sequesters more TRP-2-specific T cells than spleen ( $>4$-fold), vaccine-draining lymph nodes (VdLNs), and tumor (>18-fold) (Supplemental Figure 4C). Thus, addition of gp100/IFA vaccination to CTLA-4 blockade diverts both vaccination-induced and anti-CTLA-4-induced tumor-specific Teffs away from the tumor and toward the vaccination site.

Exhaustion and apoptosis of sequestered anti-CTLA-4-activated $C D 8^{+}$Teffs. To determine the fate of both pmel-1 and non-pmel-1 Teffs, we first analyzed Teff activation status in peripheral blood mononuclear cells (PBMCs) (Figure 3A and Supplemental Figure 3 , A and B). Next, we analyzed proliferation, function, and apoptosis markers of Teffs in PBMCs, VdLNs, and spleen, and at the tumor and vaccination sites. pmel-1 Teffs proliferated vigorously at all sites in mice receiving gp100/IFA, whereas non-pmel-1 Teffs proliferated less in mice vaccinated with gp100/IFA than in mice vaccinated with control/IFA (Figure 3B). Both pmel-1 Teffs and non-pmel-1 Teffs expressed multiple activation/checkpoint molecules including PD-1 and LAG3 (Figure 3C) (23). We next analyzed T-box transcription factors eomesodermin (Eomes) and T-bet, both major mediators of $\mathrm{T}$ cell fate and function through regulation of expression of PD-1 (24) and IFN- $\gamma$ in $\mathrm{CD}^{+}$Teffs $(25,26)$. We found downregulation of T-bet and upregulation of Eomes in both pmel-1 and non-pmel- 1 CD8 ${ }^{+}$Teffs at the vaccination site (Supplemental Figure 4D). We also found reduced secretion of IFN- $\gamma$ and TNF- $\alpha$ in TRP-2-specific Teffs (Figure 3D) and p15-specific Teffs at the vaccination site (Supplemental Figure $4 \mathrm{E})$. Thus, non-pmel-1 Teffs recruited to the vaccination site are exhausted at the level of surface markers, transcription factors, and cytokine production (27). In mice receiving peptide vaccination, the defect in cytokine production was maintained in the pool of Teffs that did reach the tumor site (Figure 3D). Teffs at the vaccination site also showed increased expression of Fas/CD95 death receptor (Figure 3E). Simultaneously, we observed a large population of $\mathrm{CD} 11 \mathrm{~b}^{+} \mathrm{Gr}-1^{+}$myeloid cells with high programmed death ligand 1 (PD-L1) and Fas ligand (FasL) expression at the gp100 vaccination site (Supplemental Figure $4 \mathrm{~F}$ ). Teffs at the vaccination site and in the VdLNs showed unchanged expression of prosurvival Bcl-xL and proapoptotic Bim, but reduced expression of the prosurvival protein Bcl-2 (Supplemental Figure 4G) and a high degree of apoptotic death (Figure 3F). Together, these data indicate that pmel-1 and non-pmel-1 Teffs become sequestered at the gp100/ IFA vaccination site, where they undergo proliferation, functional exhaustion, and eventual elimination through apoptotic death.

CXCR3 is required for $C D 8^{+}$Teff localization to tumor. Teff migration from blood into peripheral tissues, including tumors, is regulated by interactions between chemokine receptors on Teffs and their ligands expressed in target tissues. We explored the molecular mechanism of $\mathrm{CD}^{+}$Teff accumulation at the tumor and vaccination sites, possibly pointing to avenues of shifting Teff accumulation from vaccination site to tumor site. To this end, we evaluated chemokine receptor and ligand expression (28-30) following therapy with anti-CTLA-4 and vaccination (Figure 4A). Among a repertoire of chemokines including $4 \mathrm{C}-\mathrm{C}$ motif receptors (CCR4, CCR5, CCR6, and CCR7) and C-X-C motif receptor 3 (CXCR3) and their canonical ligands, only CXCR3 and its ligands showed concordant expression (Figure 4B and Supplemental Figure 5A). Both pmel-1 and non-pmel-1 Teffs expressed CXCR3 in blood (Figure 4B), whereas CXCR3's ligands, CXCL9 and CXCL10, were highly expressed at the tumor and vaccination sites (Figure 4C and Supplemental Figure 5B). Notably, the addition of gp100/IFA vaccination to anti-CTLA-4 therapy significantly reduced CXCL9 and CXCL10 expression in the tumor compared with anti-CTLA-4 monotherapy $(P<0.003)$. IFN- $\gamma$ levels at both the tumor and vaccination sites correlated with CXCL9 and CXCL10 levels (Figure 4C), consistent with IFN- $\gamma$ 's known ability to increase transcription of CXCL9 and CXCL10 in endothelial cells and myeloid cells $(31,32)$. Antibody blockade of CXCR3 reduced therapeutic antitumor efficacy of anti-CTLA-4-based therapy (Figure 4D), and reduced accumulation of pmel-1 and non-pmel-1 Teffs at both the tumor and vaccination sites (Figure 4E). In patients with melanoma, CXCL9 and CXCL10 expression in tumor correlated with improved overall survival, likely through the resulting infiltration of tumor-controlling $\mathrm{CD} 8^{+} \mathrm{T}$ cells (Figure 4F and Supplemental Figure 6) (33-35). Overall, Teffs induced by anti-CTLA-4 therapy and vaccination required CXCR3 to localize to both the vaccination site and the tumor site, and blockade of CXCR3 did not shift Teffs from vaccination site to tumor site.

$C D 8^{+}$Teff accumulation at vaccination and tumor sites is dependent on LFA-1/ICAM-1 interaction. While chemokines are critical for $\mathrm{T}$ cell trafficking to tissues, $\mathrm{T}$ cell entry and retention also require $\mathrm{CD}^{+}$Teff engagement with adhesion molecules, expressed as surface glycoproteins on a variety of hematopoietic and nonhematopoietic cells $(36,37)$. Since leukocyte function-associated antigen 1 (LFA-1, also known as $\alpha_{\mathrm{L}} \beta_{2}$ ) and very late antigen 4 (VLA-4, also known as $\alpha_{4} \beta_{7}$ ) were both expressed on pmel-1 Teffs and non-pmel-1 Teffs in blood (Figure 5B), we analyzed expression of their cognate ligands, intercellular adhesion molecule 1 
A

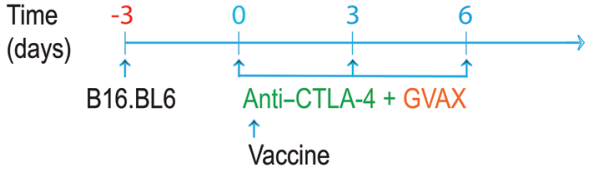

B

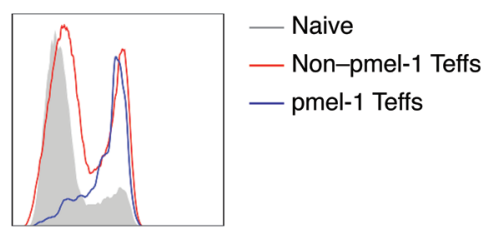

CXCR3

C

흘

$$
\text { Anti-c }
$$$$
\text { | }
$$

$0 \quad 100 \quad 2000$

Untreated $14.5 \pm 2.3$

Control/IFA B. $2 \pm 1.3$

Gp100/IFA $18 \pm 2.6$

Anti-CTLA-4

Anti-CTLA-4 + control//FA $136.5 \pm 7.9$

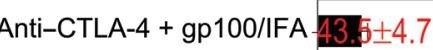

Control/IFA-2.5 \pm 0.7

Gp100/IFA-16.4 \pm 1.8

Anti-CTLA-4 + control//FA-3.9 \pm 0.7

Anti-CTLA-4 + gp100/IFA $34.8 \pm 2.7$

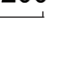

$55.5-3.1$

$-53.8+2.2$

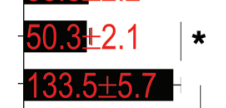

$\left|\frac{133.5 \pm 5.7}{121.2 \pm 7.8}\right|$

$-7.1 \pm 0.2$

$-18.3 \pm 2.5$

-

60

CXCL9 (ng/ml)

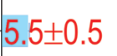

$29.4 \pm 2.4$

\begin{tabular}{lllll}
\hline $0 \quad 20$ & 40 & 0 & 0.75 & 1.5
\end{tabular}
D

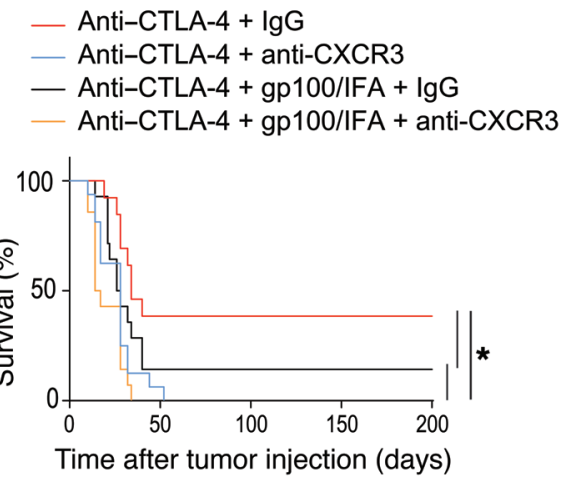

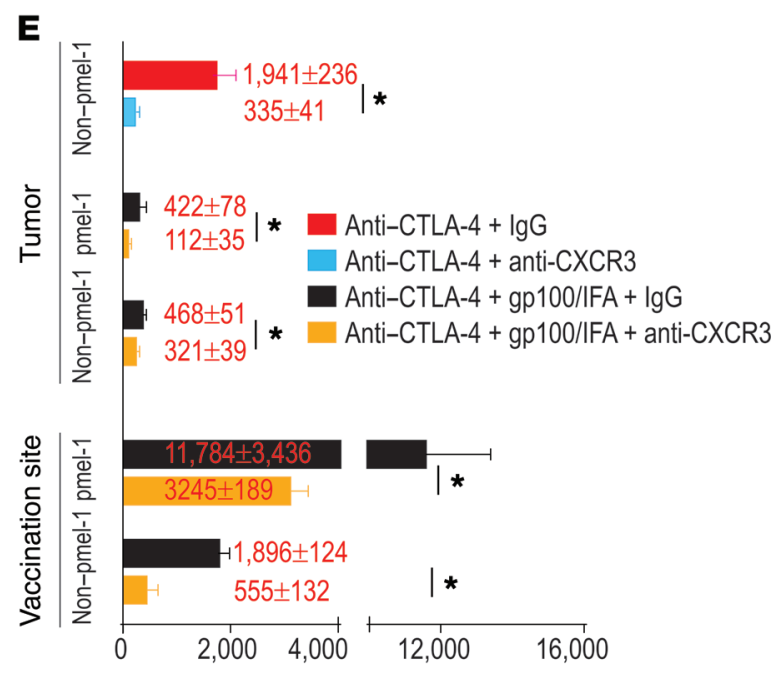

$\mathbf{F}$
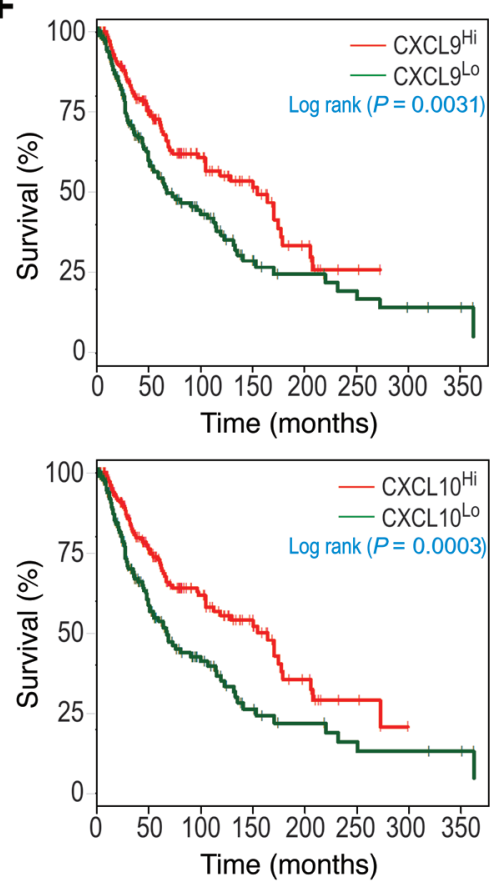
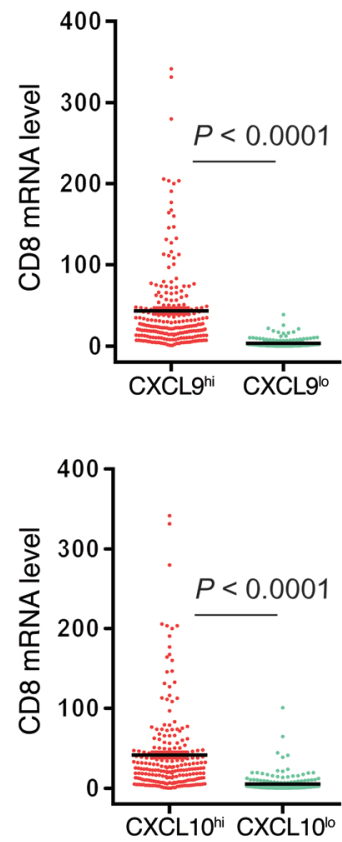

Figure 4. CXCR3 is required for CD8+ Teff localization to tumor. (A) Experimental scheme. Mice bearing 3-day-old, s.c. B16-BL6 melanomas received naive pmel-1 T cells and early anti-CTLA-4 therapy or vaccination with hgp100 in IFA. (B) Chemokine receptor expression on naive CD8 ${ }^{+}$T cells, pmel-1 CD8 ${ }^{+}$Teffs, and non-pmel-1 CD8 ${ }^{+}$Teffs in blood 9 days after vaccination. (C) Mice bearing 3-day-old, s.c. B16-BL6 melanomas received pmel-1 T cells and early antiCTLA-4 therapy or vaccination with hgp100/IFA or control/IFA. Cytokine and chemokine concentrations in supernatant from tumor and vaccination site homogenates 9 days after vaccination. All data shown are mean \pm SEM and are representative of 3 experiments $\left(n=5\right.$ mice per group, ${ }^{*} P<0.05$ determined by unpaired 2-tailed $t$ test). ( $\mathbf{D}$ and $\mathbf{E}$ ) Mice bearing 3-day-old, s.c. B16-BL6 melanomas received naive pmel-1 T cells and early anti-CTLA-4 therapy or vaccination with hgp100 in IFA (s.c.) or anti-CXCR3 (i.p.) or IgG therapy (i.p.) on days 3, 5, 7, 9, and 11 after tumor injection. (D) Kaplan-Meier survival curves. (E) Absolute number of non-pmel-1 and pmel-1 T cells (mean \pm SEM, $n=5$ ) at the tumor and vaccination sites (mean \pm SEM, $n=5$ ) analyzed 9 days after the start of CXCR3 blockade. Data shown are representative of 3 experiments. (F) CXCL9 and CXCL10 mRNA expressions by RNA sequencing and overall patient survival obtained from public TCGA repositories (https://tcga-data.nci.nih.gov and http://gdac.broadinstitute.org/). Bars show mean \pm SEM CD8 mRNA level as determined by paired 2-tailed $t$ test. 
(ICAM-1) and vascular cell adhesion molecule 1 (VCAM-1), on vasculature in the tumor and vaccination sites. Anti-CTLA-4 monotherapy markedly enhanced ICAM-1 and VCAM-1 expression by the tumor endothelium in an IFN- $\gamma$-dependent manner (Figure 5 , A and C) $(38,39)$. This enhancement was markedly blunted by addition of gp100/IFA vaccination (Figure 5C), as was tumor infiltration by pmel-1 and non-pmel-1 Teffs (Supplemental Figure 7). At the vaccination sites, treatment-induced ICAM-1 and VCAM-1 expression was not dependent on IFN- $\gamma$, lacked clear association with the vasculature (Supplemental Figure 8), and instead was abundant on SSC ${ }^{\text {hi }}$ CD $11 b^{\text {hi }}$ Ly6 $6 G^{\text {lo }}$ Ly6 $\mathrm{C}^{\text {hi }} \mathrm{F} 4-80^{+}$CCR2 ${ }^{++}$inflammatory monocytes (iMos) and $\mathrm{SSC}^{\mathrm{lo}} \mathrm{CD} 11 \mathrm{~b}^{\text {hi }} \mathrm{Ly} 6 \mathrm{G}^{\mathrm{hi}} \mathrm{Ly} 6 \mathrm{C}^{\mathrm{lo}} \mathrm{F} 4-80^{\mathrm{lo}} \mathrm{C}$ $\mathrm{CR}^{+}$granulocytic cells (Grans) (Figure 6A). To evaluate whether ICAM-1 blockade could shift Teffs from the vaccination site to the tumor site, we blocked ICAM-1 and found that therapeutic impact was significantly reduced (Figure 6B), and both pmel-1 Teffs and non-pmel-1 Teffs at the tumor and vaccination sites were reduced (Figure 6C). Interestingly, ICAM-1 blockade reduced iMos at the tumor site following anti-CTLA-4 monotherapy, and at the gp100/ IFA vaccination site in vaccinated mice, whereas Grans remain unchanged (Figure 6D). iMos expressed elevated levels of ICAM1 and VCAM-1 (Figure 6A), as well as E-selectin (CD62E) and P-selectin (CD62P) (data not shown), possibly explaining their ability to efficiently retain Teffs at the vaccination site $(40,41)$ (Figure $6 \mathrm{C}$ and Supplemental Figure 7). The Cancer Genome Atlas (TCGA) mRNA data analysis of tumor resections from patients with melanoma indicated that higher ICAM-1 expression correlated with improved survival $(P<0.01)$ and increased $\mathrm{CD}^{+} \mathrm{T}$ cell tumor infiltrate $(P<0.0001)$ (Figure 6E), supporting the notion that ICAM-1 is critical to T cell-mediated tumor rejection in mice and humans. These results indicate that LFA-1/ICAM- 1 interactions are required for the accumulation of Teffs at both the tumor and vaccination sites, and that ICAM-1 expression in the tumor site, but not in the vaccination site, is driven by IFN- $\gamma$ produced by Teffs.

CCL2 and CXCR3 mediate a feed-forward loop of CD8 ${ }^{+}$Teffs and inflammatory monocyte accumulation at the vaccination site. We found a large population of ICAM-expressing inflammatory myeloid cells, iMos (>30-fold), and Grans (>40-fold) at the vaccination site compared with the tumor site (Figure 7A). We asked whether these inflammatory myeloid cells at the vaccination site orchestrated the chemokine and cell adhesion signals required for the observed sequestration of Teffs. In the setting of CTLA-4 blockade, iMo and Gran accumulation at the vaccination site, but not the tumor site, correlated with high expression of CCL2 (Figure 7, B and C), a central chemokine for recruitment and activation of monocytes (42). Since Teff retention correlated with infiltrating iMos and Grans at the vaccination site (Figure 6, C and D), we asked whether $\mathrm{CD}^{+}$Teff recruitment is dependent on iMo and Gran recruitment to the inflamed vaccination site, in line with a recent report where neutrophils mediated recruitment of Teffs to sites of viral infection (43). In gp100/IFA-vaccinated mice, the neutralization of CCL2 reduced the numbers of CCR2 ${ }^{+}$iMos and Grans at the vaccination site, whereas recruitment to the tumor was unaffected (Figure 7D). The number of pmel-1 and non-pmel1 CCR2- Teffs (Figure 7B) at the vaccination site was reduced in mice treated with anti-CCL2 $\mathrm{mAb}$ (Figure 7E). CCL2 neutralization slightly shifted accumulation of Teffs from the vaccination site to the tumor site (Figure 7E) (44). Together, these data indicate that inflammatory myeloid cells sustain accumulation and retention of Teffs at the inflammatory vaccination site.

Next, we asked whether $\mathrm{CD}^{+}$Teffs at the vaccination or tumor site in turn promoted iMo and/or Gran accumulation, establishing a feed-forward loop of local inflammation, inflammatory myeloid cell infiltration, and $\mathrm{T}$ cell retention. Indeed, CXCR3 blockade reduced the number of CXCR3 ${ }^{+}$pmel-1 and non-pmel-1 Teffs (Figure 4E), as well as CXCR3-iMos at the vaccination site (Figure 7F and Supplemental Figure 9A), whereas Gran numbers remained unchanged (Figure 7G). Simultaneously, skin inflammation at the vaccination site was reduced (Supplemental Figure 9B). Interestingly, CXCR3-mediated Teff trafficking to the vaccination site diverted iMos from trafficking to the tumor site and instead resulted in their accumulation at the vaccination site (Figure 7F).

$\mathrm{CXCR}^{+} \mathrm{T}$ cells at the vaccination site induced myeloid cell recruitment (Figure 4E and Figure $7 \mathrm{~F}$ ), which was also CCL2 dependent (Figure 7, D and E), and IFN- $\gamma$ expression correlated with expression of both CXCL9 and CXCL10 (Figure 4C) and CCL2 (Figure 7C), so we next studied whether IFN- $\gamma$ at the vaccination site directly contributed to the recruitment of myeloid cells (Figure 7A). Skin injected with recombinant IFN- $\gamma$ emulsified in IFA, but not skin injected with IFA alone, became infiltrated with iMos, suggesting that $\mathrm{T}$ cell-derived IFN- $\gamma$ directly induces iMo recruitment to IFA vaccination sites (Supplemental Figure 10). Interestingly, Gran accumulation was induced by IFA independently of IFN- $\gamma$ (Supplemental Figure 10). Together, these data suggest that IFN- $\gamma$ release from Teffs in response to vaccine antigen recruits iMos to the IFA vaccination site. iMos and other stromal cells are induced to produce chemokines, drawing in more $\mathrm{T}$ cells, which through IFN- $\gamma$ release draw in more iMos, in a vicious cycle of myeloid- and T cell-driven inflammation.

Nonpersistent viral vaccine synergizes with therapeutic CTLA-4 and PD-L1 checkpoint blockade. Clinically used gp100/IFA vaccine formulation is poorly biodegradable and forms a persistent antigen depot in vivo $(13,14)$. Since we here observed that IFA-based vaccination also sequestered anti-CTLA-4-induced CD8 ${ }^{+}$Teffs, with specificities unrelated to the vaccine antigen, we tested whether these deleterious effects could be overcome by using a different, nonpersistent vaccine formulation. Immunization of mice with DCs pulsed with gp100 peptide significantly increased tumor regression and tumor-free survival after anti-CTLA- 4 treatment (47\%) compared with mice receiving anti-CTLA-4 monotherapy (13\%), DCs pulsed with irrelevant chicken OVA peptide plus antiCTLA-4 (0\%), DCs pulsed with gp100 peptide (13\%), DCs pulsed with OVA (0\%), or no treatment (0\%) (Supplemental Figure 11, A-C; $P<0.0001)$. Thus, anti-CTLA-4-induced tumor destruction was abrogated by vaccination with gp100 peptide in IFA, but enhanced by vaccination with DCs pulsed with gp100 peptide. Similarly, vaccination with vesicular stomatitis virus encoding gp100 (VSV.gp100) synergized with anti-CTLA-4 therapy, resulting in a significantly greater tumor-free survival rate $(53 \%)$ compared with treatment with anti-CTLA-4 (22\%), anti-CTLA-4 and gp100/IFA (8\%), or VSV.gp100 alone (13\%) $(P<0.0001$, Figure 8 , $\mathrm{A}$ and $\mathrm{B})$. Since PD-L1 blockade is another major checkpoint blockade therapy for patients with cancer, we also tested vaccination in a setting of PD-L1 blockade. We observed that anti-PD-L1 therapy 
A

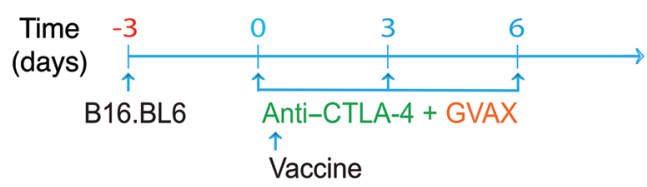

B

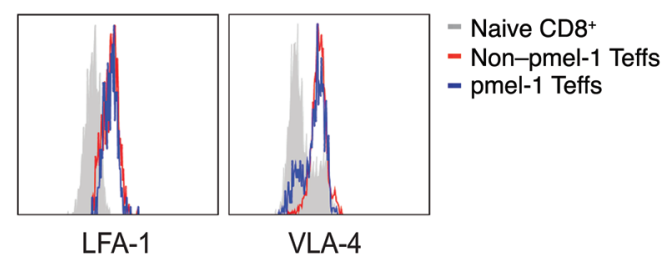

C
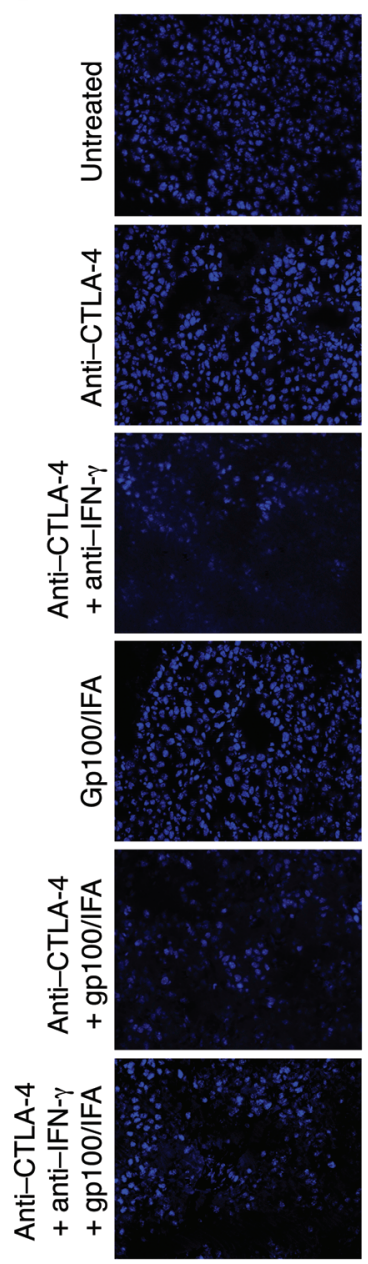

DAPI

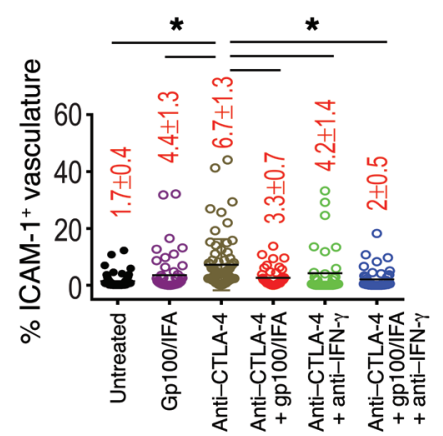

CD31
Tumor
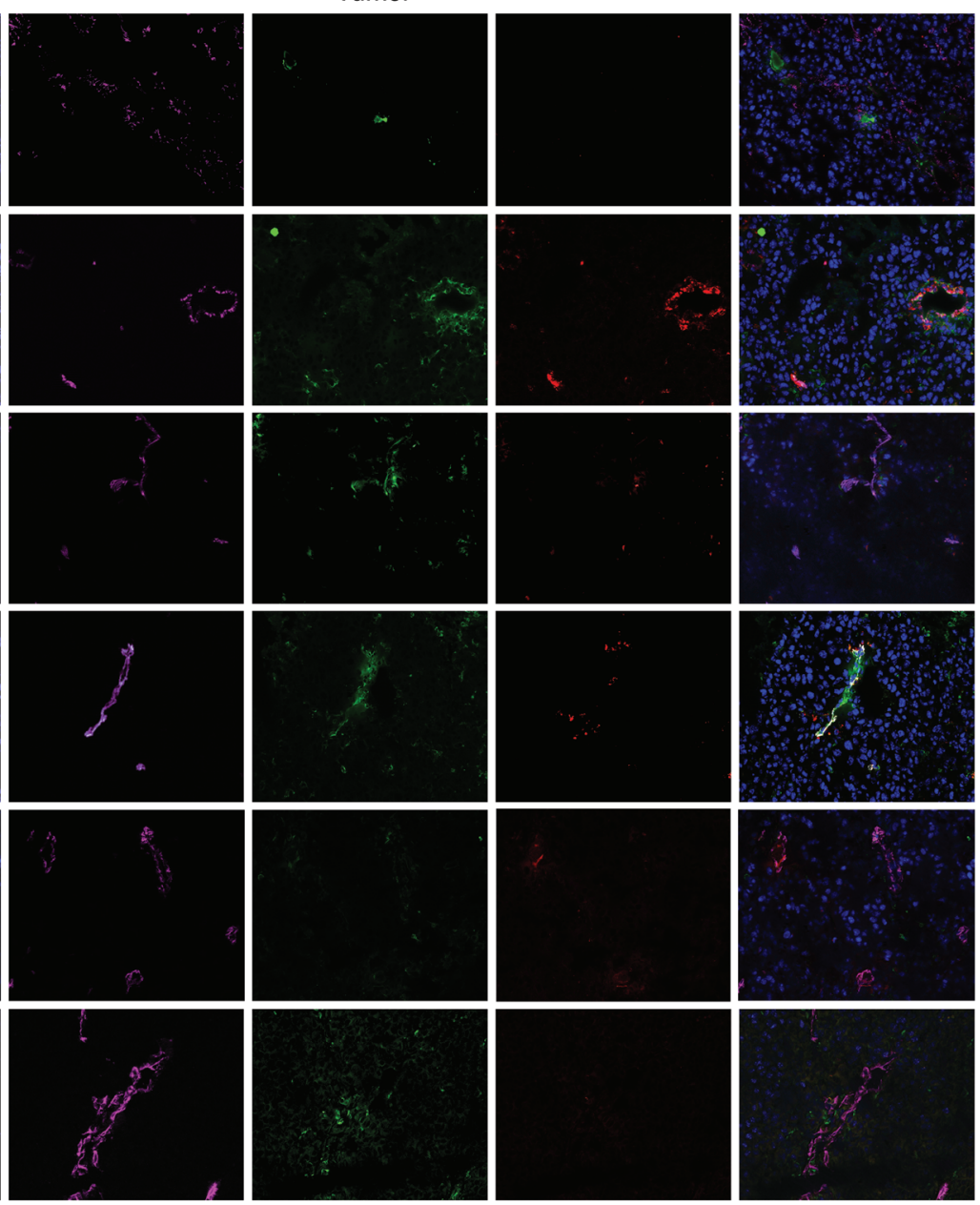

ICAM-1

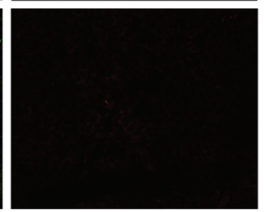

VCAM-1

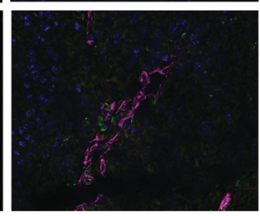

All

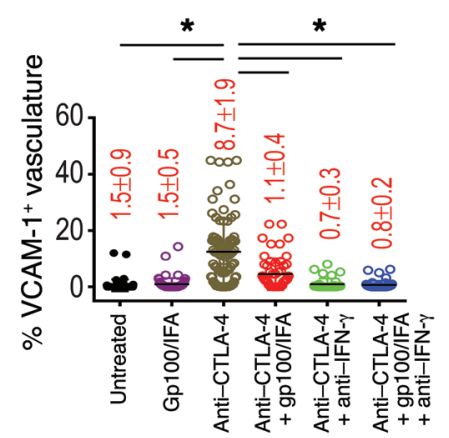

Figure 5. Impact of vaccination on the ICAM-1/VCAM-1 expression on tumor vasculature. (A) Experimental scheme. (B) Expression of adhesion surface markers on naive, pmel-1, and non-pmel-1 CD8 ${ }^{+}$T cells in PBMCs 9 days after vaccination, anti-CTLA-4 therapy, or left untreated. (C) Mice bearing B16-BL6 melanomas were injected i.v. with pmel-1 T cells. Mice were immunized with hgp100 or control in IFA on day 3; and/or received anti-CTLA-4 therapy on days 3, 6, and 9; and/or were neutralized with IFN- $\gamma$ on days 3, 5, 7, 9, and 11 after tumor injection; or they were left untreated. Graphs show ICAM-1 or VCAM-1 expression on CD31+ vasculature at tumor site 9 days after vaccination. Data are mean \pm SEM and are representative of 2 experiments ( $n=5$ mice per group, ${ }^{*} P<0.05$ determined by nonparametric Kruskal-Wallis test). 
was also impaired by gp100/IFA vaccination; however, vaccination with VSV.gp100 strongly synergized with PD-L1 blockade, curing $50 \%$ of the mice (Figure $8 \mathrm{C}$ ). Thus, vaccine formulation directly controlled the ability of vaccination to synergize with the CTLA-4 or PD-L1 checkpoint blockade. As before (Figure 4E), CXCR3 mediated the accumulation of both gp100-specific and TRP-2-specific CD8 ${ }^{+}$Teffs at the tumor site and gp100/IFA vaccination site in this setting of virus-based vaccination (Supplemental Figure 12). Remarkably, the addition of gp100/IFA abrogated the efficacy of VSV.gp100 vaccination, sharply reducing intratumoral Teff numbers and animal survival (Supplemental Figure 12). Regardless of vaccine formulation, pmel-1 Teffs were found in multiple tissues, but only gp100/IFA, and not gp100/saline or VSV.gp100, vaccination recruited large numbers of pmel-1 Teffs to the vaccination site (Supplemental Figure 13). Over time, gp100/IFA vaccination resulted in progressively reduced peripheral blood Teff levels, proliferation, trafficking, and IFN- $\gamma$ secretion (Supplemental Figure 14, A-D). Thus, T cell sequestration after gp100/IFA vaccination is a dominant effect that can abrogate effective checkpoint blockade monotherapy, or even effective combination therapy with checkpoint blockade and an otherwise effective, nonpersistent (viral) vaccine.

Synergy between anti-CTLA-4 therapy and VSV.gp100 was also impaired in genetically ICAM-1-deficient mice (Supplemental Figure 15, A and B). ICAM-1 antibody blockade decreased tumor infiltration of Teffs, and in the presence of persistent gp100/IFA vaccine, a markedly higher number of functional Teffs accumulated in the blood (Supplemental Figure 15, C and D) (45), likely due to their inability to exit the blood into target tissues. Taken together, the results indicate that ICAM-1/LFA- 1 interaction is critical for $\mathrm{CD}^{+}$Teff accumulation in the tumor, regardless of vaccine formulation.

Nonpersistent vaccine formulations overcome primary resistance to CTLA-4 and PD-L1 checkpoint blockade therapy. Viral vaccines form one of several classes of nonpersistent anticancer vaccine formulations. It is therefore possible that the difference in therapeutic synergy between virus-based and IFA-based vaccination were not due to the latter's establishment of a persistently inflamed vaccination site, but to unique immunogenic attributes of the viral vaccine. To directly establish the importance of antigen formulation, we compared vaccination with the persistent gp100/IFA vaccine formulation to vaccination with a nonpersistent, water-based gp100 peptide formulation in combination with anti-CTLA-4 in mice with more advanced (7-day), established melanoma (Figure 9A). Since water-based peptide vaccines do not efficiently prime $\mathrm{T}$ cell responses, we supplemented both the water-based and IFA-based vaccine with a combination of immunostimulatory molecules (agonistic anti-CD40 mAb, the TLR7 agonist imiquimod, and IL-2, collectively named covax) (13). After receiving the nonpersistent, water-based vaccine formulation, $21 \%$ of mice were cured compared with $6 \%$ of mice cured after vaccination with gp100/IFA ( $P<0.0006$, Figure 9B). We also tested synergy with PD-L1 checkpoint blockade therapy (46) and found that nonpersistent, water-based gp100 peptide vaccine activity synergizes with anti-PD-L1 blockade. In fact, $44 \%$ of mice receiving anti-PD-L1 and gp100/saline treatment were cured (>200 days) compared with mice receiving anti-CTLA-4 and gp100/saline (24\%), anti-PD-L1 (0\%), anti-CTLA-4 (0\%), or gp100/saline monotherapy (0\%) (Figure 9C). Similar synergy was seen between PD-L1 blockade and viral vaccination, whereas IFA-based vaccination significantly blunted therapeutic efficacy of PD-L1 blockade (Figure 9D).

Finally, we tested persistent versus nonpersistent vaccine formulations in combination with dual CTLA-4/PD-L1 checkpoint blockade therapy, which is currently the most potent therapy for patients with metastatic melanoma (2). In a challenging setting of 7-day established tumors where dual checkpoint blockade cured only $10 \%$ of the mice, addition of nonpersistent, viral gp100 vaccination resulted in the cure of $67 \%$ of the mice $(P<0.0001$, Figure 9E). Conversely, dual checkpoint blockade with gp100/IFA vaccination did not cure any mice, and gp100/IFA vaccination completely destroyed the therapeutic efficacy of VSV.gp100 vaccination plus checkpoint blockade (Figure 9E).

\section{Discussion}

The introduction of CTLA-4 and PD-1 checkpoint blockade therapy is a milestone in cancer treatment, yet clinical benefit is limited to a subset of patients $(1,3,47-51)$. Based on their mechanisms of action, CTLA- 4 and PD-1 blockades are most effective in the presence of preexisting tumor-specific $\mathrm{T}$ cell responses (9, 52-54). However, due to poor immunogenicity of many tumors, such $\mathrm{CD} 8^{+} \mathrm{T}$ cell-mediated antitumor immunity is often weak and insufficient. Vaccination could induce expansion and diversification of the tumor-specific $\mathrm{T}$ cell pool, and in this regard, recent progress has been made in clinical cancer vaccine formulations, delivery, and ability to potentiate anticancer immune response (55-59). There exists a great variety of cancer vaccine formulations, each with its unique advantages (60). Clinical trials using DC vaccine (61), synthetic long peptides (62), DNA vaccine (63), RNA vaccine $(64)$, whole-tumor cell vaccine $(65,66)$, and viral vaccine (67) have shown efficacy in inducing tumor-specific T cell responses, sometimes with clinical benefit. However, robust clinical evidence of increased efficacy of checkpoint blockade therapy in combination with vaccination is lacking. Here we show that peptide vaccine based on IFA, thus far the most commonly used experimental cancer vaccine formulation for combination with checkpoint blockade, failed to synergize with anti-CTLA-4 and anti-PD-L1 checkpoint blockade therapy.

Specifically, we found that tumor-specific Teffs, induced by checkpoint blockade therapy, were diverted away from the tumor by specific chemokines and adhesion molecules when a concurrent vaccine was given at a distant site, resulting in reduced antitumor activity of the checkpoint blockade therapy (Figure 10). Remarkably, these therapy-induced, tumor-specific Teffs had specificities other than the gp100 vaccine antigen, yet became sequestered at the gp100 vaccination site, where they eventually became dysfunctional and underwent apoptosis. Sequestration was mediated by engagement of chemokine receptors and adhesion molecules including CXCR3 and ICAM-1. CXCR3 and ICAM-1 controlled optimal localization of anti-CTLA-4-induced tumor-specific Teffs to both tumor and vaccination sites, and their blockade was unable to shift Teffs from the vaccination site to the tumor site. Neutralization of IFN- $\gamma$, a $\mathrm{CD}^{+} \mathrm{T}$ cell effector cytokine and inducer of ICAM- 1 and CXCR3 ligands, actually reduced 
A

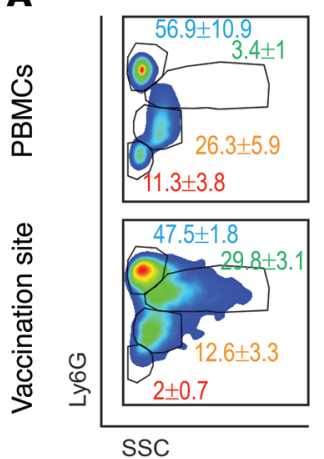

B

- Anti-CTLA-4 + $\lg G$

- Anti-CTLA-4 + anti-ICAM-1

- Anti-CTLA-4 + gp100/IFA + IgG

- Anti-CTLA-4 + gp100/IFA + anti-ICAM-1

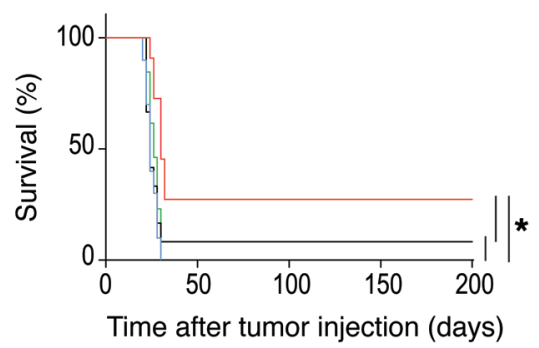

D

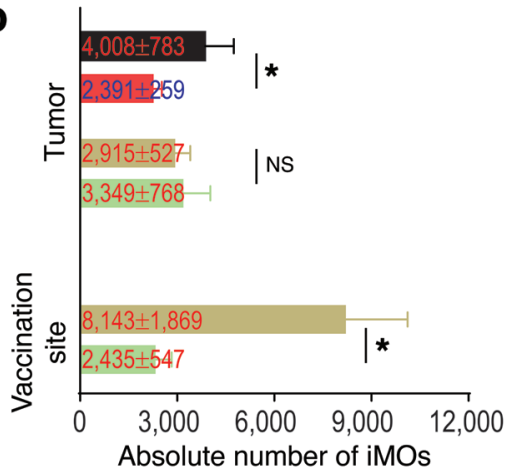

C

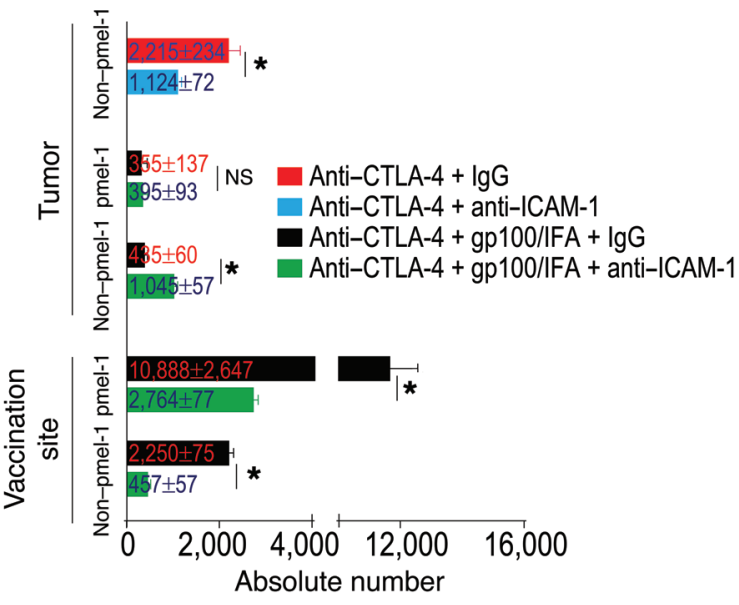

Absolute number

CCR2
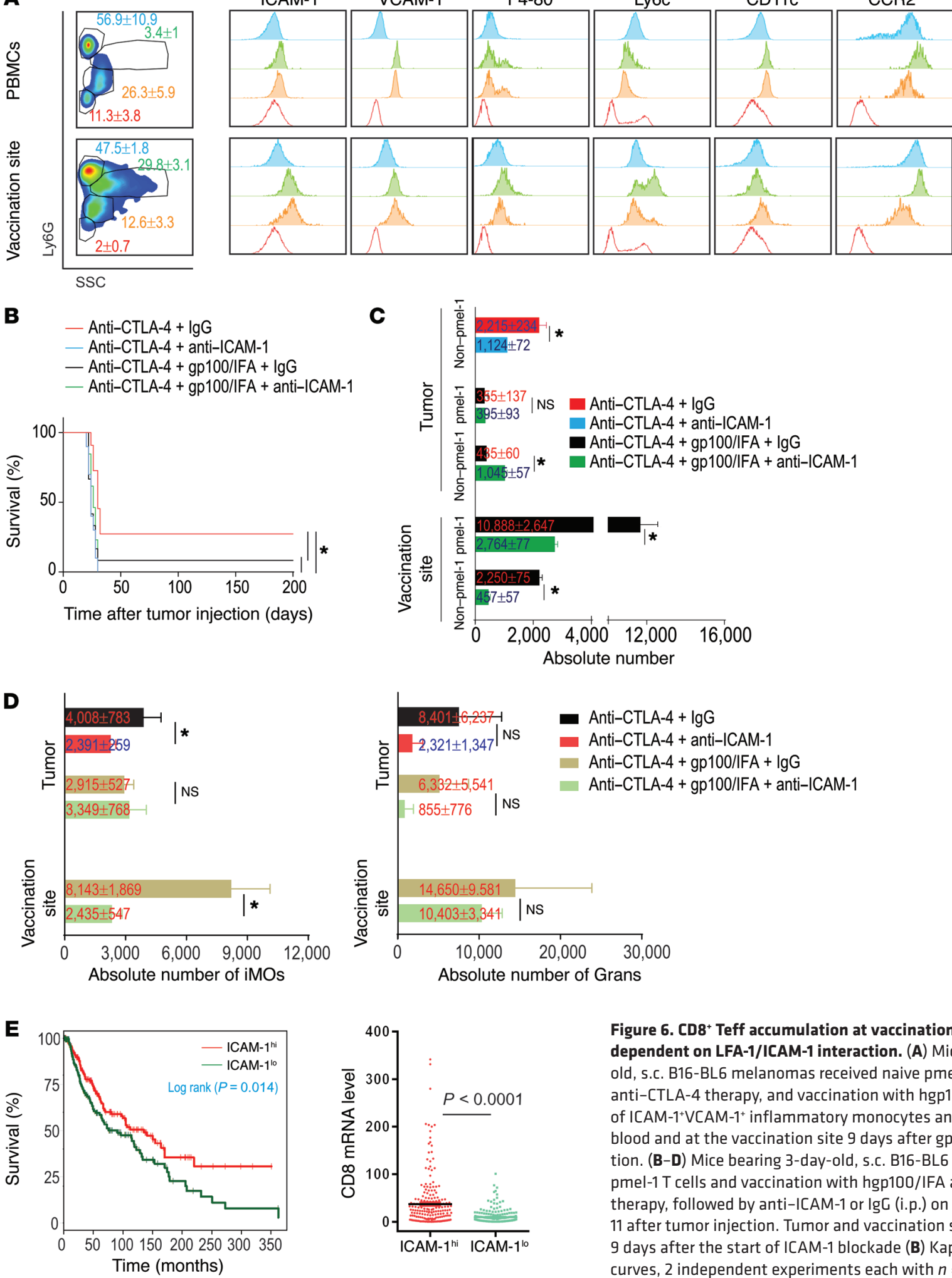

Figure 6. $\mathrm{CDB}^{+}$Teff accumulation at vaccination and tumor sites is dependent on LFA-1/ICAM-1 interaction. (A) Mice bearing 3-dayold, s.c. B16-BL6 melanomas received naive pmel-1 T cells, early anti-CTLA-4 therapy, and vaccination with hgp100 in IFA. Presence of ICAM-1+VCAM-1+ inflammatory monocytes and granulocytes in blood and at the vaccination site 9 days after gp100/IFA vaccination. (B-D) Mice bearing 3-day-old, s.c. B16-BL6 tumors received i.v. pmel-1 T cells and vaccination with hgp100/IFA and/or anti-CTLA-4 therapy, followed by anti-ICAM-1 or IgC (i.p.) on days 3, 5, 7, 9, and 11 after tumor injection. Tumor and vaccination sites were analyzed 9 days after the start of ICAM- 1 blockade (B) Kaplan-Meier survival curves, 2 independent experiments each with $n=10$ mice per group. ${ }^{*} P<0.05$, log-rank test. (C) Non-pmel- 1 and pmel-1 CD8 ${ }^{+}$Teffs at the tumor and vaccination sites. Data are mean \pm SEM, $n=5$, ${ }^{*} P<0.05$ as determined by unpaired 2-tailed $t$ test. (D) iMos and Grans at the tumor and vaccination sites. Plots are mean $\pm \mathrm{SEM}, n=5$, ${ }^{*} P<0.05$ as determined by unpaired 2 -tailed $t$ test. Data shown (B-D) are representative of 2 experiments. (E) ICAM-1 mRNA expression by RNA sequencing and overall patient survival obtained from public TCGA repositories (https://tcga-data.nci.nih.gov and http://gdac.broadinstitute.org/). 
$\mathrm{CD}^{+}$Teff accumulation at the tumor, shifting Teffs to the vaccination site. Our findings serve to illustrate that the expression of ICAM-1 and CXCR3 ligand molecules is crucial to facilitate Teff adhesion in both the tumor and other inflamed tissues, including vaccination sites. IFN- $\gamma$-induced expression of ICAM- 1 and CXCR3 ligands is also markedly increased and required for therapeutic efficacy after anti-CTLA-4 monotherapy. Consequently, neutralization of these molecules is not a viable strategy to redistribute Teffs from the vaccination site to the tumor.

The presence of immune cells in human melanomas correlated with the expression of defined chemokine subsets $(29,68)$. Analysis of 225 primary melanomas (35) revealed overexpression of CXCR3 ligand molecules CXCL9 and CXCL10 around a high density of tumor high endothelial venules. Indeed, CXCR3 expression on tumor-infiltrating, activated $\mathrm{T}$ cells is considered a good prognostic marker and is associated with improved survival in patients with melanoma $(34,69)$ and patients with advanced serous ovarian cancer (70), suggesting that CXCL9 and CXCL10 dominantly control the localization of such activated, potentially tumoricidal T cells. TCGA data on stage I-IV human melanoma samples confirm that mRNA levels of CXCR3, ICAM, and IFN- $\gamma$ in tumors also strongly correlate with enhanced patient survival, and with the presence of $\mathrm{CD}^{+} \mathrm{T}$ cells. In contrast, analysis of cellular infiltrates in skin resections from vaccine injection sites in patients with melanoma following vaccination with multiple peptides in IFA showed accumulation of $\mathrm{CXCR}^{+} \mathrm{CD} 8 \mathrm{~T}$ cells, with minimal IFN- $\gamma$ production $(14,68,71)$.

Together, our data are consistent with a scenario where circulating Teffs infiltrate tumor tissue or the vaccination site and encounter cognate antigen, causing the local release of IFN- $\gamma$, which increases expression of chemokines such as CXCL9 and CXCL10 and adhesion molecules such as ICAM-1 by host cells. Increased expression of CXCL9, CXCL10, and ICAM-1 in turn attracts additional $\mathrm{CXCR}^{+}$Teffs, supporting a vicious cycle of $\mathrm{T}$ cell infiltration, cytokine secretion, and local inflammation. When the vaccine is persistently present, as in the case of gp100 peptide emulsified in nonbiodegradable IFA, this process results in chronic sequestration of Teffs at the vaccination site (13). Importantly, non-gp100-specific Teffs induced by CTLA-4 checkpoint blockade also become sequestered through CXCR3 and ICAM-1 engagement.

At the IFA vaccination site, these detrimental effects on Teffs appear to be further compounded through a distinct pattern of cooperative, chemokine-linked, feed-forward trafficking involving Teffs, iMos, and Grans. CXCR3-dependent Teff accumulation at the vaccination site drove expression of CCL2 and influx of inflammatory myeloid cells expressing ICAM-1 and VCAM-1, driving both additional $\mathrm{T}$ cell accumulation and inflammation in a feed-forward loop. $(31,72,73)$. It is possible that this same feedforward cooperation involving (a) the link between CXCL9/10 and CCL2, and (b) LFA-1/ICAM-1-mediated cell-cell adhesion could explain why Teffs favor the vaccination site over the tumor site, which has a paucity of myeloid cells when a distant inflamed vaccination site is present. A recent study of experimental autoimmune encephalomyelitis (EAE) demonstrated that autoreactive $\mathrm{T}$ cells withstand the sheer force of the cerebrospinal fluid and maintain their attachment to leptomeninges through LFA-1- and VLA- 4-mediated cell-cell adhesion to leptomeningeal macrophages; this interaction was further potentiated by CXCR3 signaling and antigenic stimulation (74). Thus, under inflammatory conditions there appears to be an interdependent recruitment of Teffs and iMos to inflamed tissues (75). Gran recruitment to the vaccination site, on the other hand, appears to be independent of Teff recruitment, as evidenced by minimal impact of CXCR3 blockade-mediated reduction in $\mathrm{T}$ cell accumulation on their recruitment. CCL2 depletion reduced the number of Teffs and iMos trapped at the vaccination site, which interestingly also modestly shifted Teffs from vaccination site to tumor site. Several studies have demonstrated chemokine-mediated, cooperative trafficking of $\mathrm{T}$ cells and innate immune cells $(31,72,73)$. In patients with melanoma, $\mathrm{CXCR}^{+} \mathrm{T}$ cells have also been reported to preferentially accumulate at sites of IFA-based vaccination (14). Thus, the dynamics of chemokine-linked, feed-forward cooperation among inflammatory immune cell subsets reported in the current study provide mechanistic insight into the observed increased accumulation of Teffs and inflammatory myeloid cells at cutaneous vaccination sites in mice and possibly in patients with cancer $(68,71)$.

Cooperation between CD8 ${ }^{+}$Teffs and iMos is increasingly regarded as a key process in several inflammation-driven human disease models $(43,75,76)$. In patients with chronic obstructive pulmonary disease (COPD), inflammatory macrophages and $\mathrm{CD}^{+} \mathrm{T}$ cells showed enhanced migration into CXCR3 and CCR5 chemokines, leading to inflammation of the lung parenchyma and the airways (76). In patients with diabetes, $C D 8^{+}$Teffs infiltrating adipose tissue exacerbated the inflammatory reaction by inducing accumulation and activation of macrophages (75). Our data suggest that a similar process also promotes detrimental $\mathrm{T}$ cell sequestration at the inflamed vaccination site after IFA-based vaccination. Mechanistically, we find that several critical elements of the molecular machinery of chemokines and integrins that control $\mathrm{T}$ cell trafficking are shared between the tumor and vaccination sites, and hence these are not suitable targets for shifting Teffs from vaccination site to tumor site. Instead, preventing the formation of a persistent, chronically inflamed vaccination site by altering vaccine formulation offers a direct path toward the design and use of vaccines that synergize with checkpoint blockade therapy.

IFA-based, long-term persisting vaccines continue to be widely used in clinical trials of cancer vaccines. However, less persistent vaccine formulations are gaining in popularity, including water-based, cellular, viral, and nucleic acid-based formulations. We previously demonstrated the utility of a water-based peptide vaccine, and here extend those findings with DC and virus-based vaccines, demonstrating marked therapeutic activity and profound synergy with checkpoint blockade. Thus, we envision a shift from long-term, persisting antigen depot vaccines to quickly resolving, short-term depot vaccines for the induction of therapeutic anticancer $\mathrm{T}$ cell responses.

Overall, our results indicate that IFA-based vaccination induces an inflamed vaccination site that recruits, functionally impairs, and eventually destroys tumor-specific Teffs induced by anti-CTLA- 4 checkpoint blockade therapy through a mechanism dependent on inflammatory monocytes, CCL2, IFN- $\gamma$, CXCR3, and ICAM-1, causing reduced tumor control. Nonpersistent vaccine formulations can reverse these undesir- 
A

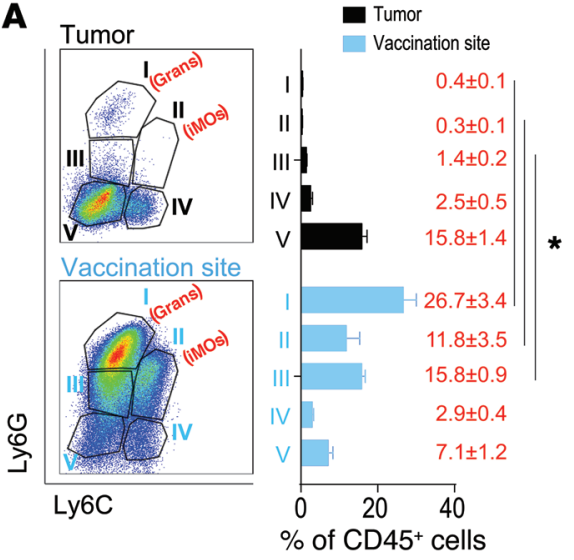

B

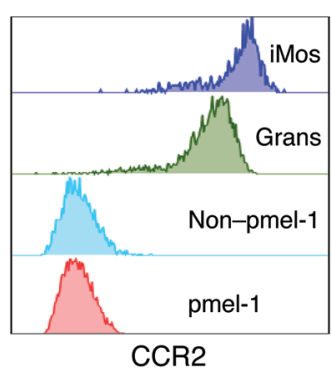

E

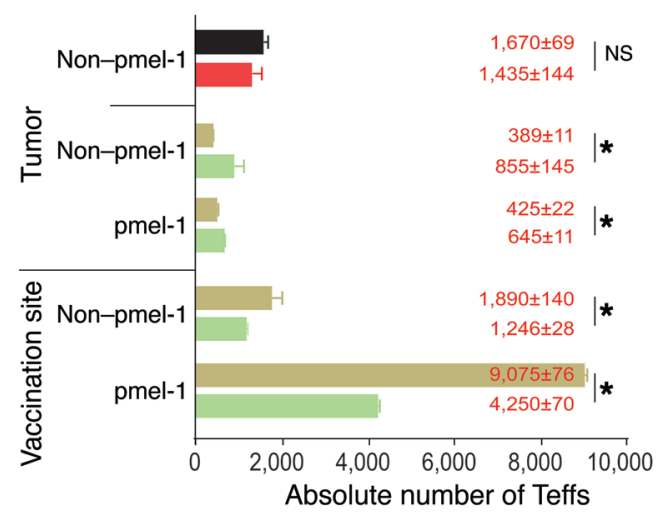

C

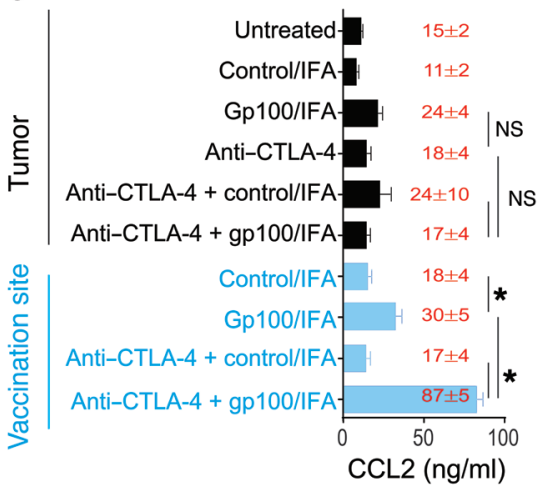

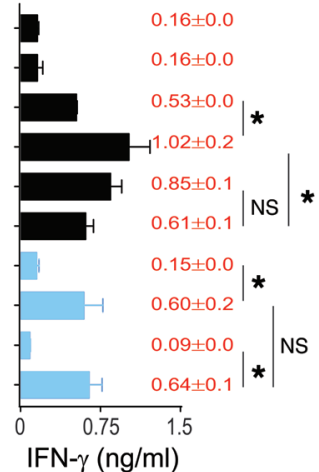

IFN- $\gamma(\mathrm{ng} / \mathrm{ml})$
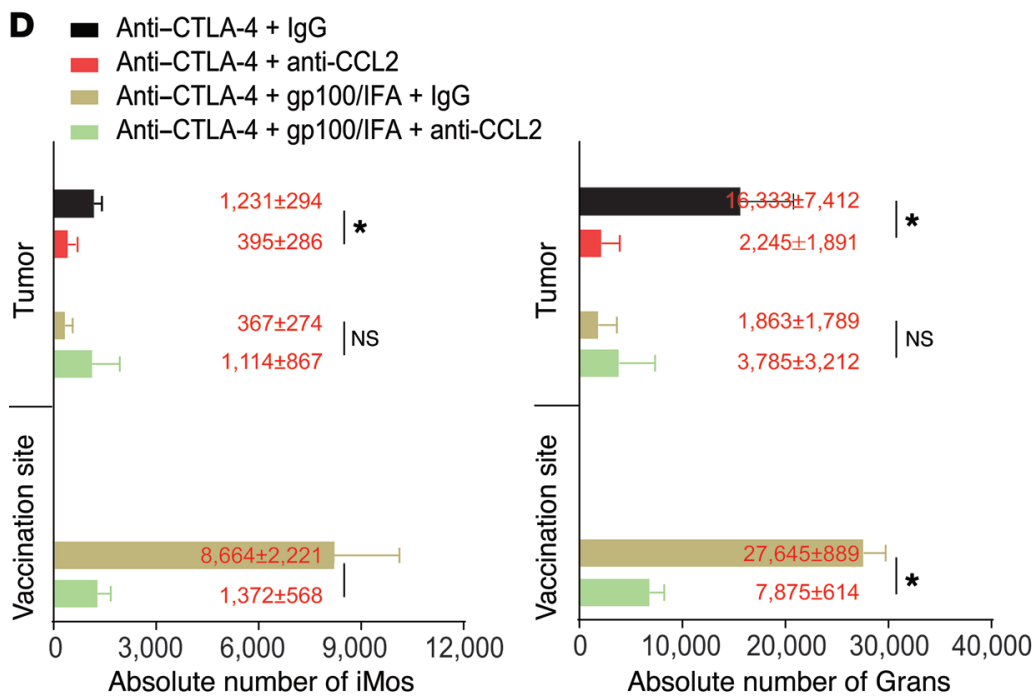

$\mathbf{F}$

$$
\begin{aligned}
& \text { Anti-CTLA-4 + IgG } \\
& \text { Anti-CTLA-4 + anti-CXCR3 } \\
& \text { Anti-CTLA-4 + gp100/IFA + IgG } \\
& \text { Anti-CTLA-4 + gp100/IFA + anti-CXCR3 }
\end{aligned}
$$
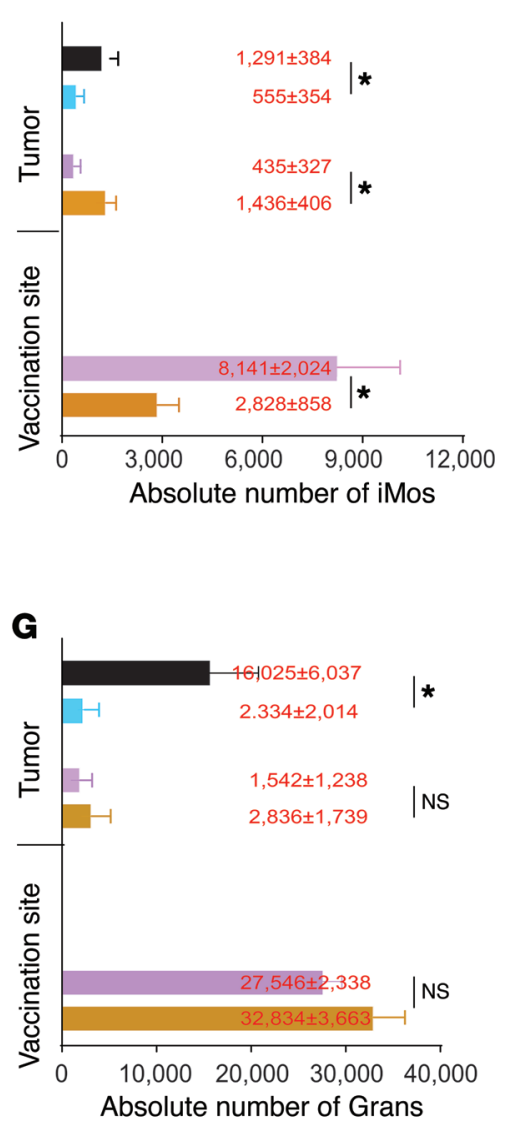

Figure 7. CCL2 and CXCR3 mediate a feed-forward loop of CD8 ${ }^{+}$Teffs and inflammatory monocyte accumulation at the vaccination site. (A and $B$ ) Mice bearing 3-day-old, s.c. B16-BL6 melanomas were injected with naive pmel-1 T cells, vaccinated with hgp100/IFA, and received early anti-CTLA-4 therapy. (A) Distribution of CD11b+ leukocyte subsets at tumor and vaccination sites. (B) CCR2 expression on leukocyte subsets as in $\mathbf{A}$ and CD8 ${ }^{+}$non-pmel-1 and pmel-1 Teffs in blood observed 9 days after vaccination. (C) Mice bearing 3-day-old, s.c. B16-BL6 melanomas received pmel-1 T cells and early anti-CTLA-4 therapy or vaccination with hgp100/IFA or control/IFA. CCL2 and IFN- $\gamma$ chemokine concentrations (mean \pm SEM) in supernatant from tumor and vaccination site homogenates 9 days after vaccination. Data shown are representative of 3 experiments, each with $n=5$ mice per group. ${ }^{*} P<0.05$ as determined by unpaired 2-tailed $t$ test. ( $\mathbf{D}$ and $\mathbf{E}$ ) Mice treated the same as in $\mathbf{C}$ underwent CCL2 depletion or received IgG on days 3, 5, 7, 9, and 11 after tumor injection. (D) Absolute number of iMos and Grans at the tumor and vaccination sites. (E) Absolute number of CD8 $8^{+}$Teffs at the tumor and vaccination sites. (F and C) Mice treated as in C received anti-CXCR3 mAb therapy or IgC on days 3, 5, 7, 9, and 11 after tumor injection. Plots shown are absolute number of (F) iMos and (C) Grans at the tumor and vaccination sites. Data shown are mean \pm SEM, representative of 3 experiments, each with $n=5$ mice per group. ${ }^{*} P<0.05$ as determined by unpaired 2-tailed $t$ test. 


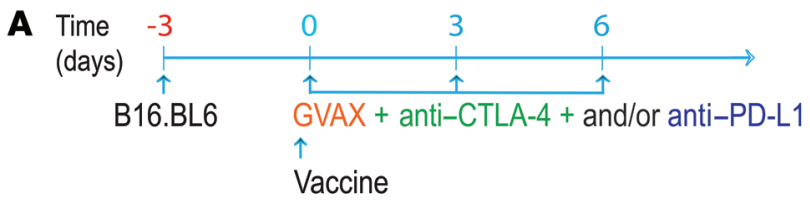

B
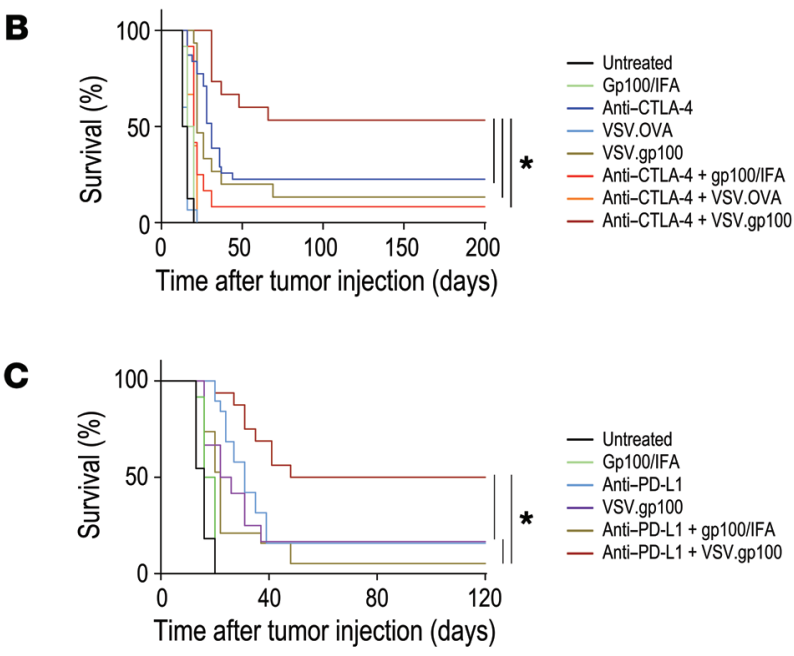

able effects and synergize with anti-CTLA-4 and anti-PD-L1 checkpoint blockade, overcoming primary resistance to checkpoint blockade therapy and greatly enhancing complete cure rates. These results provide a direct explanation for the puzzling lack of synergy between anti-CTLA-4 therapy and gp100/IFA vaccination in patients with melanoma. They also highlight the importance of vaccine formulation in future clinical trials of combination therapy with checkpoint blockade and vaccination and point the way to increase the therapeutic efficacy of checkpoint blockade for patients with cancer.

\section{Methods}

Mice and tumor cells. pmel-1 TCR transgenic mice on a C57BL/ 6 background (The Jackson Laboratory) were crossed with CD90.1 congenic mice to yield pmel- $1^{+/+} \times \mathrm{CD} 90.1^{+/+}$mice (herein referred to as pmel-1 mice). C57BL/6J-Tyr-2J/J albino, OT-I TCR transgenic, and ICAM-1knockout mice were purchased from The Jackson Laboratory. B16-BL6 melanoma cells and granulocyte-macrophage colony-stimulating factor (GM-CSF) producing B16-BL6 were cultured in DMEM supplemented with $1 \mu \mathrm{g} / \mathrm{ml}$ penicillin, $1 \mathrm{mg} / \mathrm{ml}$ streptomycin, $50 \mathrm{mg} / \mathrm{ml}$ gentamicin, 2 mM L-glutamine, and 8\% FCS (Invitrogen). E.G7.OVA cells were maintained in the presence of G418 (EMD Millipore). B16.white is a gp100 nonpigmented B16 variant maintained in RPMI 1640 with $10 \%$ heatinactivated FBS, $0.03 \%$ L-glutamine, $100 \mu \mathrm{g} / \mathrm{ml}$ streptomycin, and 100 $\mu \mathrm{g} / \mathrm{ml}$ penicillin. Retroviral supernatant production and $\mathrm{T}$ cell transduction were performed as previously detailed (77).

Treatment. Mice were shaved on the back and received $100 \mu \mathrm{l}$ PBS (s.c., $2.5 \times 10^{4} \mathrm{~B} 16 / \mathrm{BL} 6$ melanoma cells). Mice were treated (s.c., posterior costal region) on days 3,6 , and 9 with $1 \times 10^{6}$ irradiated (160 Gy) B16-BL6 producing GM-CSF (Gvax) together with $3 \times 200 \mu \mathrm{g}$ $(10 \mathrm{mg} / \mathrm{kg}), 100 \mu \mathrm{g}(5 \mathrm{mg} / \mathrm{kg})$, and $100 \mu \mathrm{g}(5 \mathrm{mg} / \mathrm{kg})$ anti-CTLA-4 (clone 9H10, Bioxcell) or anti-PD-L1 (clone 10F.9G2, Bioxcell) i.p. on days 3,6 , and 9 , which constituted early treatment. In the late treatment approach, mice received Gvax treatment with anti-CTLA-4 or
Figure 8. Nonpersistent viral vaccine synergizes with therapeutic CTLA-4 and PD-L1 checkpoint blockades. (A) Experimental scheme. Kaplan-Meier survival curves in mice with 3-day-old, s.c. B16 melanomas after receiving naive pmel-1 T cells (i.v.). (B) Early anti-CTLA-4 therapy and vaccination with hgp100/IFA (s.c.), VSV.gp100 (i.v.), VSV.OVA, or combination. (C) Early anti-PD-L1 (i.p.) plus Gvax therapy and vaccination with hgp100/IFA (s.c.), VSV.gp100 (i.v.), or combination. Data were pooled from 2 independent experiments, each with $n=10$ mice per group. ${ }^{*} P<0.05$, log-rank test.

anti-PD-L1 on days 7, 10, and 13 after tumor injection. On days 3, 5, 7, 9, and 11, some mice received (i.p.) $100 \mu \mathrm{l}, 5 \times 200 \mu \mathrm{g}(10 \mathrm{mg} / \mathrm{kg})$ anti-ICAM-1, anti-CXCR3, anti-CCL2, anti-IFN- $\gamma$, or IgG. In another tumor challenge model, $5 \times 10^{5}$ E.G7.OVA cells were injected s.c. into C57BL/6 mice on day 0. Recombinant rat IFN- $\gamma$ (rIFN- $\gamma$ ) was purchased from PeproTech. Mice received s.c. injection of rIFN- $\gamma(200$ $\mathrm{ng} / \mathrm{ml}$ ) together with control/IFA.

DC generation and transfer. Bone marrow cells isolated from femurs and tibiae of C57BL/6 mice were RBC lysed and cultured in RPMI containing $10 \%$ FCS, 1 mM sodium pyruvate, HEPES, penicillin, streptomycin, and $\beta$-mercaptoethanol (EMD Millipore), supplemented with $0.1 \mu \mathrm{g} / \mathrm{ml} \mathrm{GM-CSF}$ (PeproTech). On day 3, fresh RPMI containing $0.1 \mu \mathrm{g} / \mathrm{ml} \mathrm{GM-CSF}$ was added. On day 7, nonadherent DCs were cultured in medium containing $0.1 \mu \mathrm{g} / \mathrm{ml}$ LPS for 24 hours. For transfer, DCs were pulsed for 1 hour with $10 \mu \mathrm{g} / \mathrm{ml} \mathrm{hgp} 100_{25-33}$ or chicken $\mathrm{OVA}_{257-264}$ peptides for 1 hour at $37^{\circ} \mathrm{C}$. Cells were then harvested from plates and washed 2 times in PBS, and $1 \times 10^{6}$ cells per mouse were administered through tail vein injection.

Peptide vaccination. The synthetic, high-affinity $\mathrm{H}-2 \mathrm{D}^{\mathrm{b}}$-restricted heteroclitic mouse gp100 $25-33$ peptide (hgp100, KVPRNQDWL), $\mathrm{H}-2 \mathrm{~K}^{\mathrm{b}}$-restricted peptides chicken $\mathrm{OVA}_{257-264}$ (SIINFEKL), TRP-2 ${ }_{180-188}$ (SVYDFFVWL), and p15E (KSPWFTTL) were purchased from Peptides International at a purity greater than $95 \%$. $\mathrm{H}-2 \mathrm{~K}^{\mathrm{b}}$-restricted TRP-2 pentamer (SVYDFFVWL) was purchased from ProImmune. Mice received 1,000 naive pmel-1 or OT-I T cells i.v. and were vaccinated with 2 separate s.c. injections at the base of the tail or in each flank with $100 \mu$ lof either saline or saline and IFA emulsion (1:1, vol/ vol), each containing $100 \mu \mathrm{g}$ hgp $100_{25-33}$ or $50 \mu \mathrm{g} \mathrm{OVA}_{257-264}$ peptide. In the early treatment approach, mice were vaccinated on day 3 after tumor injection at the posterior flank with 2 injections of $100 \mu \mathrm{l}$ IFA emulsions containing $100 \mu \mathrm{g}$ hgp $100_{25-33}$ peptide, or without peptide (control), or mice were infected i.v. with $100 \mu$ l of $1 \times 10^{7} \mathrm{PFU} / \mathrm{ml} \mathrm{VSV}$. gp100 or VSV.OVA. In the late-treatment approach, mice were vaccinated on day 7 after tumor injection at the posterior flank with 2 injec- 

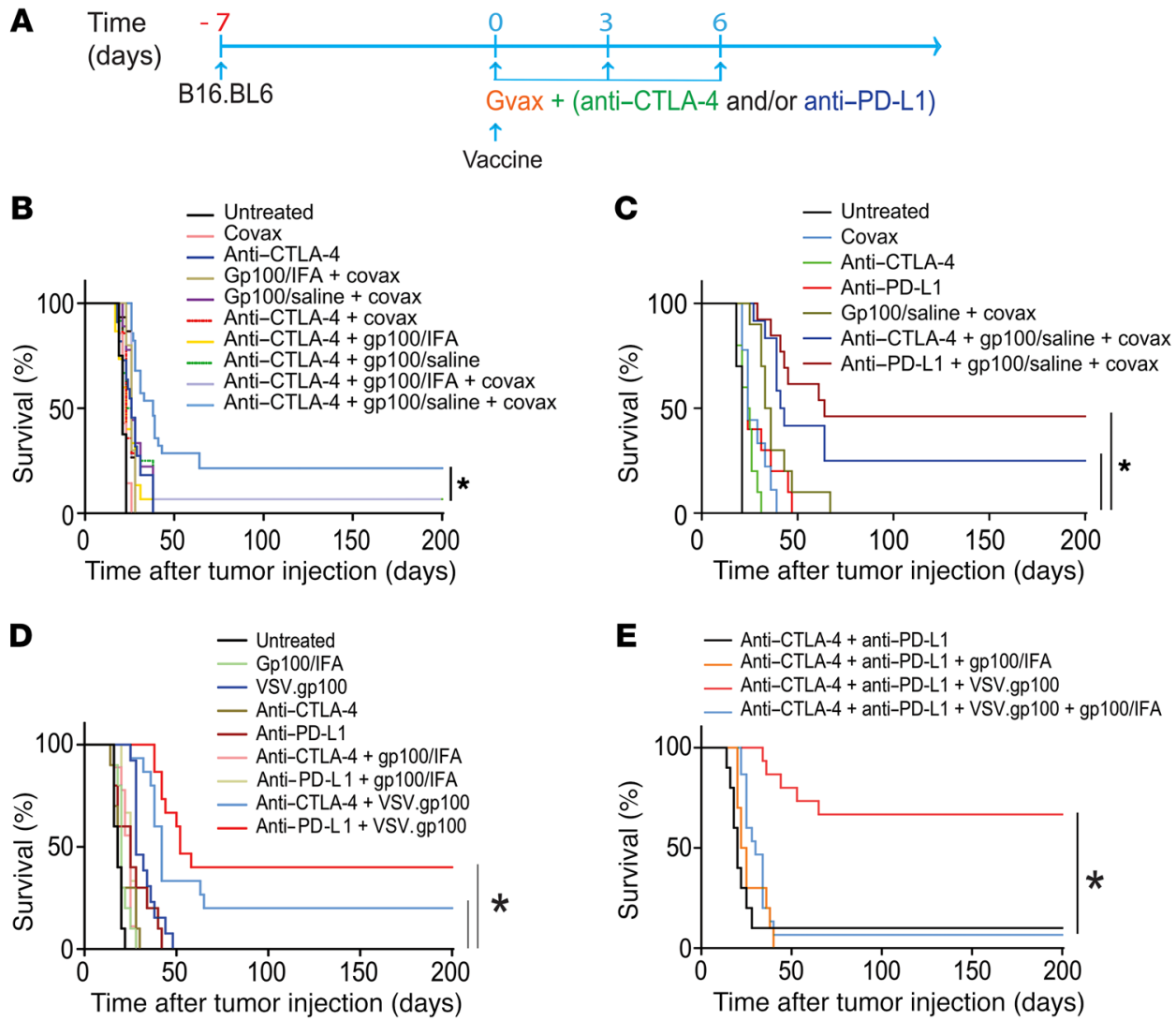

Figure 9. Nonpersistent vaccine formulations overcome primary resistance to CTLA-4 and PD-L1 checkpoint blockade therapy. (A) Experimental scheme. Kaplan-Meier survival curves of mice bearing 7-day-old, s.c. B16 tumors after receiving naive pmel-1 T cells (i.v.). (B) Late anti-CTLA-4 therapy and/or vaccination with hgp100/IFA (s.c.) or hgp100 peptide in saline (s.c.) with covax. (C) Covax or late anti-CTLA-4 or anti-PD-L1 therapy and/or hgp100 peptide in saline (s.c.) and covax, or no treatment. (D) Late anti-CTLA-4 or anti-PD-L1 therapy and/or vaccination with hgp100 peptide in IFA or VSV.gp100, or left untreated. (E) Late dual checkpoint blockade therapy with anti-CTLA-4 and anti-PD-L1 combined with vaccination with hgp100/IFA (s.c.) and/or VSV.gp100 (i.v.). Data are pooled from 2 independent experiments, each with $n=15$ mice per group. ${ }^{*} P<0.05$, log-rank test.

tions of $100 \mu \mathrm{l}$ IFA emulsions containing $100 \mu \mathrm{g}$ hgp100 ${ }_{25-33}$ peptide, or mice received covax (13) consisting of $100 \mu \mathrm{l}, 1 \times$ s.c., $500 \mu \mathrm{g} / \mathrm{ml}$ of CD40-specific mAb (clone FGK4.5, Bioxcell), and $25 \mathrm{mg}, 1 \times$ topical imiquimod cream $5 \%$ (Aldara, Fougera), and a total $100 \mu$ l, $5 \times$ i.p., $1 \times$ $10^{6} \mathrm{IU} / \mathrm{ml}$ rhIL-2 (TECIN, Hoffman La Roche Inc.) on days 0, 1, and 2.

$T$ cell imaging studies. C57BL/6J-Tyr-2J/J albino mice were injected with $3 \times 10^{5} \mathrm{~B} 16$.white cells on the shaved back. This combination of white mice and nonpigmented tumor reduces photon absorption by the skin and tumor pigment during $\mathrm{T}$ cell imaging (77). Seven days later, mice were vaccinated with $100 \mu \mathrm{l}$ saline in IFA (control) or with IFA emulsions containing $100 \mu \mathrm{g}$ hgp $100_{25-33}$ peptide (s.c.); a 50:50 mixture of 6-day-cultured $1 \times 10^{6}$ pmel- $1 \mathrm{~T}$ cells and $1 \times 10^{6}$ eGFP-sorted, v-effLuc-GFP-transduced OT-1 T cells (i.v.); and they received 3 days of IL-2 treatment. We performed the reverse experiment with saline in IFA (control) or IFA emulsions containing $50 \mu \mathrm{g}$ $\mathrm{OVA}_{257-264}$ peptide (s.c.); a 50:50 mixture of 6-day-cultured $1 \times 10^{6}$ OT-1 T cells and $1 \times 10^{6}$ eGFP-sorted, v-effLuc-GFP-transduced pmel-1 T cells (i.v.); and IL-2 treatment for 3 days.

Bioluminescence imaging was performed with a cooled chargecoupled device (CCD) camera mounted in a light-tight specimen box (IVIS 200, Xenogen). For in vivo imaging, mice were given $150 \mathrm{mg} / \mathrm{kg}$ body weight (i.p.) of D-luciferin substrate (Caliper Life Sciences), anesthetized with $1 \%-3 \%$ isoflurane, and imaged 8 minutes after D-lucif- erin injection. Regions of interest from the displayed images were designated and quantified as total photon counts or photons/second (represented by color bars) using Living Image software (Xenogen).

Tissue preparation. Mice were euthanized by $\mathrm{CO}_{2}$ inhalation. PBMCs were collected by tail bleed or cardiac puncture. Spleens, lymph nodes, and skin sections from vaccination sites and tumors were harvested and stored in cold PBS (Life Technologies). To determine the absolute number of a given cell subset per tumor or vaccination site, we adjusted cell counts quantitated by flow cytometry to absolute weight of tumor and skin from vaccine injection sites. Single-cell suspensions were prepared in PBS with $10 \%$ FCS and 2 mM EDTA (Sigma-Aldrich) by mashing tissue against the surface of a $40-\mu \mathrm{m}$ cell strainer using the plunger of a 3-ml syringe (Becton Dickinson). RBC were removed using a hypotonic lysis buffer (StemCell Technologies). Skin sections were minced using a surgical blade (Becton Dickinson) and digested with collagenase (Sigma-Aldrich) for 3 hours at $37^{\circ} \mathrm{C}$ and then passed through an 18-gauge syringe (Becton Dickinson) and filtered using a $40-\mu \mathrm{m}$ cell strainer (BD Falcon). Mice were tail bled at the indicated time points.

Preparation of tissue homogenates. On day 12 after tumor injection, tumor and skin sections from the vaccination site (6-mm diameter) were surgically removed from euthanized mice. Tissue weight measurements were performed. Tissues were homogenized in $1 \mathrm{ml}$ cold PBS using a glass homogenizer. The homogenates were transferred 


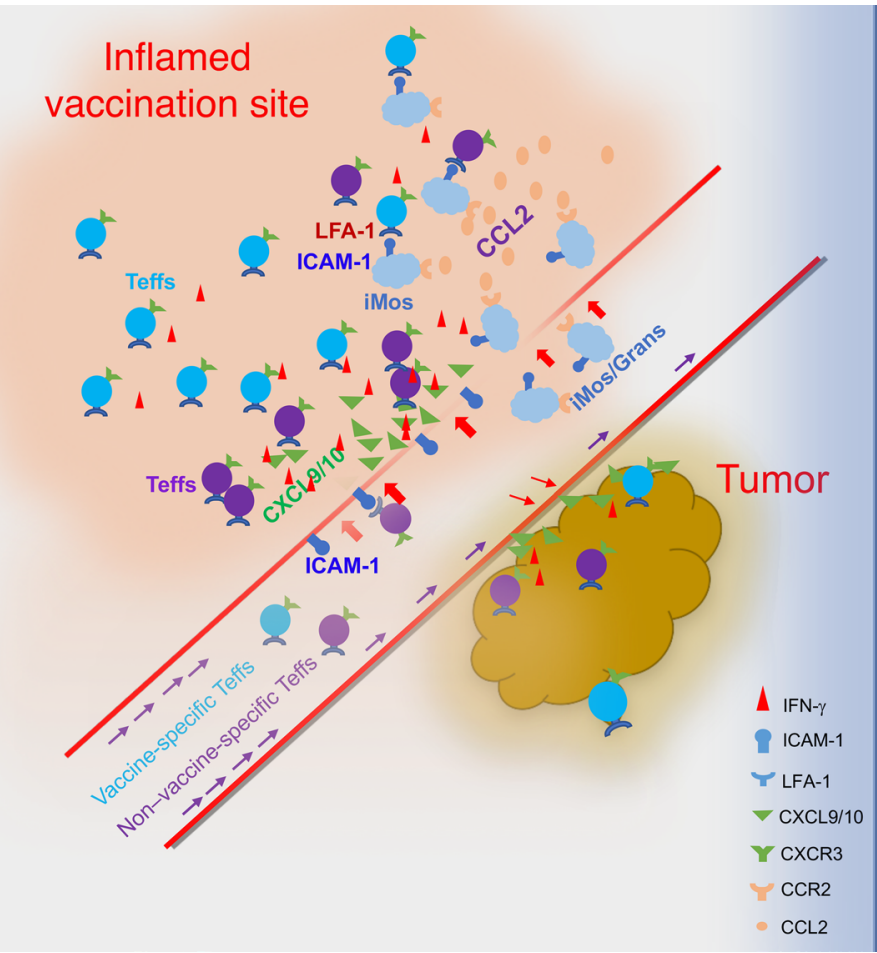

\section{Noninflamed vaccination site}

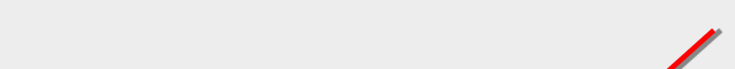

Figure 10. Mechanism of vaccine antigen-specific and non-vaccine antigen-specific Teff recruitment to the inflamed vaccination site. Left: Vaccine antigen-specific Teffs respond to vaccine antigen at the persistent vaccination site by releasing IFN- $\gamma$, which induces CCL2-dependent recruitment of ICAM+ ${ }^{+}$Mos. IFN- $\gamma$ also induces expression of $C X C L 9$ and $C X C L 10$ chemokines. CTLA- 4 blockade-induced CXCR3+LFA- $1^{\text {hi }}$ Teffs, regardless of antigen specificity, are attracted by CXCL9 and CXCL10 and engage with ICAM+ iMos in a feed-forward loop maintained by iMos, IFN- $\gamma$, CCL2, CXCL9, CXCL10, and ICAM-1. Teffs at the vaccination site do not reach the tumor and undergo apoptosis, resulting in loss of tumor control. Right: Without a persistent, inflamed vaccination site, CTLA-4 blockade-induced Teffs are free to localize to the tumor site, resulting in tumor control.

to 1.5 -ml Eppendorf tubes and centrifuged at 13,000 $\mathrm{g}$ for 10 minutes at $4^{\circ} \mathrm{C}$, and the supernatant was stored at $-80^{\circ} \mathrm{C}$ until analyzed by Luminex (Millipore) according to the manufacturer's protocol.

Histology. Tumor and skin sections dissected from a similarly treated parallel group of mice as described in the previous paragraph were snap frozen in OCT. Tissues were cut into $6-\mu \mathrm{m}$ sections then fixed with acetone/ethanol and blocked with $\mathrm{PBS} / 5 \%$ BSA, anti-CD16/32, 3\% $\mathrm{H}_{2} \mathrm{O}_{2}, 0.1 \% \mathrm{NaN}_{3}$, and Avidin/Biotin Blocking Kit (Vector Laboratories). Sections were stained with biotinylated or fluorescence-conjugated antibodies (5-20 $\mu \mathrm{g} / \mathrm{ml}$ ) to CD31 (clone 390), ICAM-1 (clone YN1/1.7.4), VCAM-1 (clone 429), CD8 $\alpha$ (clone 53-6.7), Thy1.1 (clone H1S51), and F4/80 (clone BM8) antibodies followed by peroxidase or fluorescent Streptavidin conjugates (Jackson Immunoresearch Laboratories Inc.). VCAM signals were amplified with Tyramide Signal Amplification Plus kits (PerkinElmer). Slides were mounted in SlowFade Gold (Invitrogen) and images collected on an AxioImager with Apotome (Zeiss). ImageJ (NIH) was used to analyze percent marker-positive vasculature.

TCGA analysis. TCGA mRNA expression by RNA sequencing and overall patient survival obtained from public TCGA repositories (TCGA Data Portal, https://tcga-data.nci.nih.gov and Broad Institute, http://gdac.broadinstitute.org/) were used to construct Kaplan-Meier curves for patients of all stages or with stage III cutaneous melanoma. When analyzing the additive effects of 2 genes on survival, we created 4 expression groups based on quartiles of median values.

Antibody staining and FACS analysis. Intracellular IFN- $\gamma$ staining was performed using the Cytofix/Cytoperm kit (BD Biosciences) according to the manufacturer's recommendation after 4 hours of stimulation with $1 \mu \mathrm{M}$ mouse hgp100, or TRP-2 or p15E peptides and using a 1:800 dilution of $\mathrm{mAb}$ to IFN- $\gamma$ (clone XMG1.2, BD Pharmingen). Annexin V binding assay was performed on unfixed cells with an annexin V-fluorescein isothiocyanate (FITC) staining kit according to the manufacturer's instruction (BD Biosciences). All antibodies were used at 1:200 dilution unless otherwise indicated. Antibodies used for staining included antibodies against CD8 (clone 53-6.7), CD90.1 (clone HIS51), V 22 (clone B20.1), Vß5 (clone MR9-4), CD44 (clone IM7), CD11a (clone M17/4), TNF- $\alpha$ (clone MP6-XT22), CD152 (clone UC10-4B9), CD95 Fas (clone 15A7), CD11c (clone HL3), CD11b (clone M1/70), Ly-6G (clone 1A8), CD19 (clone MB19-1), CCR7 (clone 4B12), CCR6 (clone sirx6), CCR5 (clone T21/8), $K_{\mathrm{i}}-67$ (clone SOIA15), Eomes (clone Dan11mag), T-bet (clone 4B10), CD54 (clone YN1/1.7.4), CD106 (clone 429), CD178 FasL (clone KAY10), and Ly6C (clone AL-21) (all from Affymetrix/eBioscience). Antibodies against PD-L1 (10F.9G2), LAG-3 (clone C9B7W), PD-1 (clone RMPI.30), CXCR3 (clone CXCR3-173), CD62P (clone RMP-1), CCR6 (clone 29-2L17), CCR4 (clone 2G12), and CCR2 (clone SA203G11) were all from BioLegend. Antibodies against CD178 FasL (clone KAY10), Ly-6C (clone AL-21), Vo2 (clone B20.1), and CD62E (clone 10E9.6) were all from BD Biosciences.

Statistics. All results are expressed as mean \pm SEM. Mouse and sample group sizes were $n=5$, unless otherwise indicated. Data were analyzed using paired or unpaired 2-tailed $t$ tests or nonparametric Kruskal-Wallis test where applicable, and differences were considered significant at $P<0.05$. All experiments were performed at least twice, with comparable results. Mice were euthanized when tumor size 
reached $\geq 200 \mathrm{~mm}^{2}$. Survival curves were plotted using Kaplan-Meier estimates and compared by log-rank analysis.

Study approval. Animal experiments performed in this study were approved by the Institutional Animal Care and Use Committee of the University of Texas MD Anderson Cancer Center.

\section{Author contributions}

YH, AW, TF, QH, MCN, FH, ZX, CV, HK, M Singh, M Sharma, FF, $\mathrm{DM}$, and ZD performed experiments. SMA, KSS, TF, and PS provided study materials. AW and VHE performed immunofluorescence analysis and interpreted the results. JR performed TCGA data analysis. $\mathrm{YH}$ and WWO designed experiments and wrote the manuscript.

\section{Acknowledgments}

The work was supported by the University of Texas MD Anderson Cancer Center Specialized Program of Research Excellence in Melanoma Career Development Award (P50 CA093459) to YH, and a Cancer Prevention and Research Institute of Texas (CPRIT) grant (RP140522) to WWO.

Address correspondence to: Willem W. Overwijk, Department of Melanoma Medical Oncology, University of Texas MD Anderson Cancer Center, 7455 Fannin, Unit 904, Houston, Texas 77054, USA. Phone: 713.563.5294, Email: woverwijk@ mdanderson.org.
1. Hodi FS, et al. Improved survival with ipilimumab in patients with metastatic melanoma. $N$ Engl J Med. 2010;363(8):711-723.

2. Postow MA, et al. Nivolumab and ipilimumab versus ipilimumab in untreated melanoma. N Engl J Med. 2015;372(21):2006-2017.

3. Larkin J, et al. Combined nivolumab and ipilimumab or monotherapy in untreated melanoma. N Engl JMed. 2015;373(1):23-34.

4. Topalian SL, et al. Safety, activity, and immune correlates of anti-PD-1 antibody in cancer. $N$ Engl JMed. 2012;366(26):2443-2454.

5. Gajewski TF. The next hurdle in cancer immunotherapy: overcoming the non-T-cell-inflamed tumor microenvironment. Semin Oncol. 2015;42(4):663-671.

6. Pitt JM, et al. Resistance mechanisms to immune-checkpoint blockade in cancer: tumorintrinsic and -extrinsic factors. Immunity. 2016;44(6):1255-1269.

7. Hugo W, et al. Genomic and transcriptomic features of response to anti-PD-1 therapy in metastatic melanoma. Cell. 2016;165(1):35-44.

8. Snyder A, et al. Genetic basis for clinical response to CTLA-4 blockade in melanoma. $N$ Engl J Med. 2014;371(23):2189-2199.

9. Gubin MM, et al. Checkpoint blockade cancer immunotherapy targets tumour-specific mutant antigens. Nature. 2014;515(7528):577-581.

10. Gao J, et al. Loss of IFN- $\gamma$ pathway genes in tumor cells as a mechanism of resistance to antiCTLA-4 therapy. Cell. 2016;167(2):397-404.e9.

11. Curran MA, Montalvo W, Yagita H, Allison JP. PD-1 and CTLA-4 combination blockade expands infiltrating $\mathrm{T}$ cells and reduces regulatory $\mathrm{T}$ and myeloid cells within B16 melanoma tumors. Proc Natl Acad Sci US A. 2010;107(9):4275-4280.

12. Pardoll DM. The blockade of immune checkpoints in cancer immunotherapy. Nat Rev Cancer. 2012;12(4):252-264.

13. Hailemichael Y, et al. Persistent antigen at vaccination sites induces tumor-specific $\mathrm{CD}^{+} \mathrm{T}$ cell sequestration, dysfunction and deletion. Nat Med. 2013;19(4):465-472.

14. Salerno EP, et al. Activation, dysfunction and retention of $\mathrm{T}$ cells in vaccine sites after injection of incomplete Freund's adjuvant, with or without peptide. Cancer Immunol Immunother. 2013;62(7):1149-1159.

15. Fu T, He Q, Sharma P. The ICOS/ICOSL pathway is required for optimal antitumor responses mediated by anti-CTLA-4 therapy. Cancer Res. 2011;71(16):5445-5454.

16. van Elsas A, et al. Elucidating the autoimmune and antitumor effector mechanisms of a treatment based on cytotoxic T lymphocyte antigen-4 blockade in combination with a B16 melanoma vaccine: comparison of prophylaxis and therapy. JExp Med. 2001;194(4):481-489.

17. Pilon-Thomas S, Mackay A, Vohra N, Mulé JJ. Blockade of programmed death ligand 1 enhances the therapeutic efficacy of combination immunotherapy against melanoma. JImmunol. 2010;184(7):3442-3449.

18. Overwijk WW, et al. gp100/pmel 17 is a murine tumor rejection antigen: induction of "self"-reactive, tumoricidal T cells using high-affinity, altered peptide ligand. J Exp Med. 1998;188(2):277-286.

19. Overwijk WW, et al. Tumor regression and autoimmunity after reversal of a functionally tolerant state of self-reactive CD8 ${ }^{+}$T cells. J Exp Med. 2003;198(4):569-580.

20. Hamid O, et al. A prospective phase II trial exploring the association between tumor microenvironment biomarkers and clinical activity of ipilimumab in advanced melanoma. J Transl Med. 2011;9:204.

21. Rai D, Pham NL, Harty JT, Badovinac VP. Tracking the total CD8 $\mathrm{T}$ cell response to infection reveals substantial discordance in magnitude and kinetics between inbred and outbred hosts. JImmunol. 2009;183(12):7672-7681.

22. Masopust D, Murali-Krishna K, Ahmed R. Quantitating the magnitude of the lymphocytic choriomeningitis virus-specific CD8 T-cell response: it is even bigger than we thought. J Virol. 2007;81(4):2002-2011.

23. Wherry EJ, et al. Molecular signature of CD $8^{+}$ T cell exhaustion during chronic viral infection. Immunity. 2007;27(4):670-684.

24. Kao C, et al. Transcription factor T-bet represses expression of the inhibitory receptor PD-1 and sustains virus-specific $\mathrm{CD}^{+} \mathrm{T}$ cell responses during chronic infection. Nat Immunol. 2011;12(7):663-671.

25. Szabo SJ, Sullivan BM, Stemmann C, Satoskar AR, Sleckman BP, Glimcher LH. Distinct effects of T-bet in TH1 lineage commitment and IFNgamma production in CD4 and CD8 T cells. Science. 2002;295(5553):338-342.

26. Pearce EL, et al. Control of effector $\mathrm{CD} 8^{+} \mathrm{T}$ cell function by the transcription factor eomesoder- min. Science. 2003;302(5647):1041-1043.

27. Wherry EJ, Kurachi M. Molecular and cellular insights into T cell exhaustion. Nat Rev Immunol. 2015;15(8):486-499.

28. Zlotnik A, Yoshie O. The chemokine superfamily revisited. Immunity. 2012;36(5):705-716.

29. Harlin H, et al. Chemokine expression in melanoma metastases associated with $\mathrm{CD}^{+} \mathrm{T}$-cell recruitment. Cancer Res. 2009;69(7):3077-3085.

30. Bedognetti D, et al. CXCR3/CCR5 pathways in metastatic melanoma patients treated with adoptive therapy and interleukin-2. Br J Cancer. 2013;109(9):2412-2423.

31. Luster AD, Unkeless JC, Ravetch JV. Gammainterferon transcriptionally regulates an earlyresponse gene containing homology to platelet proteins. Nature. 1985;315(6021):672-676

32. Chow MT, Luster AD. Chemokines in cancer. Cancer Immunol Res. 2014;2(12):1125-1131.

33. Mikucki ME, et al. Non-redundant requirement for CXCR3 signalling during tumoricidal T-cell trafficking across tumour vascular checkpoints. Nat Commun. 2015;6:7458.

34. Mullins IM, et al. CXC chemokine receptor 3 expression by activated CD $8^{+} \mathrm{T}$ cells is associated with survival in melanoma patients with stage III disease. Cancer Res. 2004;64(21):7697-7701.

35. Martinet L, et al. High endothelial venules (HEVs) in human melanoma lesions: major gateways for tumor-infiltrating lymphocytes. Oncoimmunology. 2012;1(6):829-839.

36. Muller WA. Transendothelial migration: unifying principles from the endothelial perspective. Immunol Rev. 2016;273(1):61-75.

37. Ley K, Laudanna C, Cybulsky MI, Nourshargh S. Getting to the site of inflammation: the leukocyte adhesion cascade updated. Nat Rev Immunol. 2007;7(9):678-689.

38. Wang X, Michie SA, Xu B, Suzuki Y. Importance of IFN-gamma-mediated expression of endothelial VCAM-1 on recruitment of CD $8^{+} \mathrm{T}$ cells into the brain during chronic infection with Toxoplasma gondii. J Interferon Cytokine Res. 2007;27(4):329-338.

39. Michailowsky V, et al. Intercellular adhesion molecule 1 deficiency leads to impaired recruitment of T lymphocytes and enhanced host susceptibility to infection with Trypanosoma cruzi. J Immunol. 2004;173(1):463-470.

40. Somersalo K, et al. Cytotoxic T lymphocytes form an antigen-independent ring junction. J Clin Invest. 2004;113(1):49-57. 
41. Chakraborty S, et al. FRET based quantification and screening technology platform for the interactions of leukocyte function-associated antigen-1 (LFA-1) with intercellular adhesion molecule-1 (ICAM-1). PLoS ONE. 2014;9(7):e102572.

42. Deshmane SL, Kremlev S, Amini S, Sawaya BE. Monocyte chemoattractant protein-1 (MCP-1): an overview. J Interferon Cytokine Res. 2009;29(6):313-326.

43. Lim K, et al. Neutrophil trails guide influenzaspecific $\mathrm{CD} 8^{+} \mathrm{T}$ cells in the airways. Science. 2015;349(6252):aaa4352.

44. Fridlender ZG, et al. CCL2 blockade augments cancer immunotherapy. Cancer Res. 2010;70(1):109-118.

45. Zumwalde NA, Domae E, Mescher MF, Shimizu Y. ICAM-1-dependent homotypic aggregates regulate $\mathrm{CD} 8 \mathrm{~T}$ cell effector function and differentiation during $\mathrm{T}$ cell activation. J Immunol. 2013;191(7):3681-3693.

46. Topalian SL, Drake CG, Pardoll DM. Immune checkpoint blockade: a common denominator approach to cancer therapy. Cancer Cell. 2015;27(4):450-461.

47. Wolchok JD, et al. Nivolumab plus ipilimumab in advanced melanoma. $N$ Engl J Med. 2013;369(2):122-133.

48. Nghiem PT, et al. PD-1 blockade with pembrolizumab in advanced Merkel-cell carcinoma. N Engl J Med. 2016;374(26):2542-2552.

49. Motzer RJ, et al. Nivolumab versus everolimus in advanced renal-cell carcinoma. $N$ Engl J Med. 2015;373(19):1803-1813.

50. Lipson EJ, et al. Tumor regression and allograft rejection after administration of anti-PD-1. NEngl J Med. 2016;374(9):896-898.

51. Borghaei H, et al. Nivolumab versus docetaxel in advanced nonsquamous non-small-cell lung cancer. N Engl J Med. 2015;373(17):1627-1639.

52. Spranger S, et al. Up-regulation of PD-L1, IDO, and $\mathrm{T}$ (regs) in the melanoma tumor microenvironment is driven by CD8 (+) T cells. Sci Transl Med. 2013;5(200):200ra116.

53. Zamarin D, et al. Localized oncolytic virotherapy overcomes systemic tumor resistance to immune checkpoint blockade immunotherapy. Sci Transl Med. 2014;6(226):226ra32.

54. Tumeh PC, et al. PD-1 blockade induces responses by inhibiting adaptive immune resistance. Nature. 2014;515(7528):568-571.

55. Fu J, et al. STING agonist formulated cancer vaccines can cure established tumors resistant to PD-1 blockade. Sci Transl Med. 2015;7(283):283ra52.

56. Bencherif SA, et al. Injectable cryogel-based whole-cell cancer vaccines. Nat Commun. 2015;6:7556.

57. Liu $\mathrm{H}$, et al. Structure-based programming of lymph-node targeting in molecular vaccines. Nature. 2014;507(7493):519-522.

58. Kim J, et al. Injectable, spontaneously assembling, inorganic scaffolds modulate immune cells in vivo and increase vaccine efficacy. Nat Biotechnol. 2015;33(1):64-72.

59. Tan AC, Goubier A, Kohrt HE. A quantitative analysis of therapeutic cancer vaccines in phase 2 or phase 3 trial. J Immunother Cancer. 2015;3:48.

60. van der Burg SH, Arens R, Ossendorp F, van Hall $\mathrm{T}$, Melief CJ. Vaccines for established cancer: overcoming the challenges posed by immune evasion. Nat Rev Cancer. 2016;16(4):219-233.

61. Kantoff PW, et al. Sipuleucel-T immunotherapy for castration-resistant prostate cancer. $N$ Engl J Med. 2010;363(5):411-422.

62. Kenter GG, et al. Vaccination against HPV-16 oncoproteins for vulvar intraepithelial neoplasia. N Engl J Med. 2009;361(19):1838-1847.

63. Trimble CL, et al. Safety, efficacy, and immunogenicity of VGX-3100, a therapeutic synthetic DNA vaccine targeting human papillomavirus 16 and $18 \mathrm{E} 6$ and $\mathrm{E} 7$ proteins for cervical intraepithelial neoplasia 2/3: a randomised, doubleblind, placebo-controlled phase $2 \mathrm{~b}$ trial. Lancet. 2015;386(10008):2078-2088.

64. Kranz LM, et al. Systemic RNA delivery to dendritic cells exploits antiviral defence for cancer immunotherapy. Nature. 2016;534(7607):396-401.

65. Le DT, et al. Safety and survival with GVAX pancreas prime and Listeria Monocytogenesexpressing mesothelin (CRS-207) boost vaccines for metastatic pancreatic cancer. JClin Oncol. 2015;33(12):1325-1333.

66. Keenan BP, Jaffee EM. Whole cell vaccines-past progress and future strategies. Semin Oncol. 2012;39(3):276-286.

67. Gulley JL, et al. Immune impact induced by PROSTVAC (PSA-TRICOM), a therapeutic vaccine for prostate cancer. Cancer Immunol Res. 2014;2(2):133-141.

68. Harris RC, et al. The vaccine-site microenvironment induced by injection of incomplete Freund's adjuvant, with or without melanoma peptides. J Immunother. 2012;35(1):78-88.

69. Messina JL, et al. 12-Chemokine gene signature identifies lymph node-like structures in melanoma: potential for patient selection for immunotherapy? Sci Rep. 2012;2:765.

70. Bronger $\mathrm{H}$, et al. CXCL9 and CXCL10 predict survival and are regulated by cyclooxygenase inhibition in advanced serous ovarian cancer. Br JCancer. 2016;115(5):553-563.

71. Schaefer JT, et al. Dynamic changes in cellular infiltrates with repeated cutaneous vaccination: a histologic and immunophenotypic analysis. J Transl Med. 2010;8:79.

72. Mantovani A, Allavena P, Sica A, Balkwill F. Cancer-related inflammation. Nature. 2008;454(7203):436-444.

73. von Andrian UH, Mackay CR. T-cell function and migration. Two sides of the same coin. $N$ Engl $J$ Med. 2000;343(14):1020-1034.

74. Schläger C, et al. Effector T-cell trafficking between the leptomeninges and the cerebrospinal fluid. Nature. 2016;530(7590):349-353.

75. Nishimura S, et al. CD $8^{+}$effector T cells contribute to macrophage recruitment and adipose tissue inflammation in obesity. Nat Med. 2009;15(8):914-920.

76. Costa C, et al. Enhanced monocyte migration to CXCR3 and CCR5 chemokines in COPD. Eur Respir J. 2016;47(4):1093-1102.

77. Rabinovich BA, et al. Visualizing fewer than 10 mouse $\mathrm{T}$ cells with an enhanced firefly luciferase in immunocompetent mouse models of cancer. Proc Natl Acad Sci U S A. 2008;105(38):14342-14346. 